Ricerca in Psicoterapia / Research in Psychotherapy 2010; 1 (13): 61-91. http://www.researchinpsychotherapy.net

\title{
Aspetti teorico-metodologici e analisi dei dati nella ricerca in psicoterapia. Parte I: La ricerca sull'esito e la ricerca sul processo - dagli anni '50 agli anni '80
}

\author{
Omar C.G. Gelo ${ }^{1,2}$, Andrea F. Auletta ${ }^{1}$ e Diana Braakmann ${ }^{2}$
}

Sommario: La psicoterapia può essere descritta facendo riferimento a due dimensioni principali: l'esito (ovvero gli effetti di un trattamento) e il processo (ovvero ciò che accade nel corso di un trattamento). Nelle sue prime fasi, lo studio empirico della psicoterapia si è concentrato principalmente, anche se non esclusivamente, su questi due aspetti distinti, dando origine a ciò che prende il nome di ricerca sull'esito e ricerca sul processo psicoterapeutico. In questo periodo, che va dai primi anni '50 ai primi anni '80, nasce e si afferma quello che può essere definito il paradigma classico della ricerca in psicoterapia, caratterizzato da una adesione ai principi metodologici della scienza neo-positivistica. Con il presente articolo intendiamo descrivere le caratteristiche principali della ricerca sull'esito e sul processo terapeutico nel corso di queste tre decadi, con particolare riferimento ai diversi approcei e disegni di ricerca, ai livelli di osservazione, e all'analisi dei dati. Inoltre, ci proponiamo di discutere quelli che noi consideriamo essere gli aspetti critici in ciascuno di questi due diversi ambiti di ricerca.

Parole chiave: ricerca in psicoterapia; ricerca sull'esito; ricerca sul processo; metodologia; analisi dei dati.

\footnotetext{
${ }^{1}$ Università del Salento; ${ }^{2}$ Sigmund Frend University Vienna.

Corrispondenza: omar.gelo@sfu.ac.at - via M. Stampacchia - 73100, Lecce
}

Ricevuto: 14 Marzo 2010 - Revisionato: 29 Aprile 2010 - Accettato: 16 Maggio 2010 
Ricerca in Psicoterapia / Research in Psychotherapy 2010; 1 (13): 61-91. http://www.researchinpsychotherapy.net

\section{Introduzione}

Nel leggere di psicoterapia si incontrano spesso i termini esito terapentico e processo terapeutico. Mentre il primo rappresenta il risultato (o l'efficacia) di un trattamento, il secondo riguarda invece ciò che accade nel corso del trattamento. Lo studio di queste due dimensioni della psicoterapia si è realizzato, almeno inizialmente, attraverso la ricerca sull'esito (o sull'efficacia) e la ricerca sul processo (Dazzi, 2006). La ricerca sull'esito intende valutare "se la psicoterapia funziona", studiando l'entità del cambiamento dei pazienti in seguito a uno o più trattamenti psicoterapeutici. Al contrario, la ricerca sul processo si propone di valutare "cosa accade in psicoterapia", attraverso lo studio del comportamento ${ }^{1}$ del paziente e/o del terapeuta nel corso delle sedute.

Il presente articolo si propone di descrivere gli aspetti essenziali della ricerca sull'esito e sul processo terapeutico relativamente al periodo che va dai primi anni '50 ai primi anni '80. Queste tre decadi testimoniano, infatti, il sorgere e il consolidarsi di quello che potremmo definire il paradigma classico della ricerca in psicoterapia che, sotto l'influenza di una visione neo-positivistica della scienza (Corbetta, 2003; Loose, 2009), si propone di applicare il metodo scientifico allo studio empirico della psicoterapia, con il fine di valutare in modo il più obiettivo possibile gli aspetti oggetto di indagine (per maggiori dettagli sullo sviluppo storico della ricerca in psicoterapia si veda Orlinsky \& Russell, 1994; Migone, 2006). La ricerca sull'esito e sul processo verranno inizialmente presentate con riferimento ai disegni di ricerca, ai livelli di osservazione delle variabili, e all'analisi dei dati. Successivamente, verranno proposte alcune considerazioni critiche di carattere teorico-metodologico e relative all'analisi dei dati.

\section{La ricerca sull'esito terapeutico}

L'esito terapentico viene tradizionalmente definito come il risultato che uno o più trattamenti producono su uno o più soggetti sottoposti a quel trattamento, e consiste così con l'indagine degli effetti della psicoterapia osservati al suo termine. Lo studio dell'esito consiste dunque, tradizionalmente, con la valutazione dell'entità della modificazione di determinate variabili - considerate essere indici o correlati del benessere clinico di un individuo - a seguito di un trattamento psicoterapeutico.

Una prima forma di studio dell'esito terapentico può essere rintracciata già nel lavoro di Freud che, nel trattamento di Anna O. (Brener \& Freud, 1892-95), descrive in che modo specifici interventi quali l’ipnosi e l’interpretazione siano efficaci nel ridurre la sintomatologia della paziente. ${ }^{2}$ Nello stesso periodo, in ambito comportamentista Watson \& Raynor (1920) presentano il primo esperimento sul caso singolo in cui viene indotta nel povero Albert quella che oggi chiameremmo sindrome da ansia

\footnotetext{
${ }^{1}$ In questo contesto, il termine 'comportamento' va inteso in senso lato, e fa riferimento a qualunque fenomeno, avvenimento e/o attività del paziente e/o del terapeuta che ha luogo all'interno della seduta. Include dunque sia aspetti relativi alla comunicazione verbale (ciò che viene detto e come) e non verbale (postura, mimica facciale, ecc.) tra paziente e terapenta, sia ad aspetti relativi al vissuto del paziente e/o del terapenta relativamente a quanto è avvenuto nel corso della seduta.

${ }^{2}$ Barlow \& Hersen (1984) suggeriscono come questo approccio sia analogo allo studio del caso singolo con baseline multipla.
} 
Ricerca in Psicoterapia / Research in Psychotherapy 2010; 1 (13): 61-91. http://www.researchinpsychotherapy.net

generalizzata, e la Jones (1924a, 1924b) dimostra che questi stati d'ansia possono essere efficacemente trattati attraverso un ri-condizionamento - che oggi chiameremmo psicoterapia dell'inibizione reciproca (Wolpe, 1958). Sempre nel corso degli anni '20, si assiste a un tentativo di valutare l'efficacia di psicoterapie psicoanalitiche (Knight, 1941) ed eclettiche (Huddleson, 192\%). I risultati di questi studi, che utilizzavano descrizioni talvolta sommarie e statistiche descrittive per analizzare $i$ dati, verranno presi in considerazione da una rassegna critica di Eysenck (1952), che giungerà alla conclusione che gli effetti della psicoterapia possono essere considerati analoghi alla remissione spontanea. Tale affermazione provocatoria segna la nascita della moderna ricerca sull'esito in psicoterapia che, a partire di primi anni '50, cercherà di dimostrare l'infondatezza dellaffermazione di Eysenck attraverso lo sviluppo e l'applicazione di metodologie sempre più sistematiche e sofisticate per lo studio dell'efficacia della psicoterapia. Le prime risposte all'affermazione di Eysenck (1952) arrivano, tra gli altri, da Rosenzweig (1954) che, oltre a sottolineare i limiti metodologici presenti nel lavoro di Eysenck, produce i primi risultati che sostengono in maniera convincente l'efficacia della psicoterapia. Gli studi di Sloane (Sloane, Staples, Cristol, Yorkstone, \& Whipple (1975) e Smith (Smith \& Glass, 19\%\%; Smith, Glass, \& Miller, 1980) produrranno risultati analoghi, introducendo inoltre delle innovazioni metodologiche degne di rilievo. In particolare, Smith \& Glass (197\%) faranno uso per la prima volta della meta-analisi, una nuova forma di analisi dei dati che permetterà da lì in poi di riassumere e valutare $\mathrm{i}$ risultati presenti nella letteratura sull'efficacia terapeutica in modo preciso e attendibile. Infine, la ricerca sull'esito verrà sempre più affiancata dalla ricerca comparata sull'esito (ad esempio Luborsky, Singer, \& Luborsky, 1975), che intende studiare l'efficacia relativa di due o più trattamenti diversi.

Allo stato attuale, vi è unanime consenso circa l'efficacia della terapia. Al contrario, il dibattito è ancora acceso circa l'efficacia relativa dei trattamenti. Se da una parte, infatti, viene sostenuto che nessuna forma di psicoterapia è più efficace di un'altra ${ }^{3}$ (ad esempio, Wampold et al., 199\%; si veda anche Lambert \& Ogles, 2004; Wampold, 2001), dall'altra sembra esserci evidenza empirica che certe forme di trattamento possano essere più efficaci di altre nel trattamento di specifici disturbi (ad esempio, CritsChristoph, 199\%; per una discussione critica si veda Lambert \& Archer, $2007)$.

Approccio e disegni di ricerca. Gli approcei di ricerca sono quello sperimentale e/o quasi-sperimentale, che nella ricerca in psicoterapia iniziano ad essere sistematicamente utilizzati dai primi anni '50. Entrambi hanno come obiettivo quello di identificare eventuali rapporti di causa effetto tra una variabile indipendente (il trattamento) e una variabile dipendente (ad esempio, la sintomatologia dei pazienti) misurata al passare del tempo (ad esempio, tra l'inizio e la fine della terapia). Inoltre, entrambi gli approcei implicano il confronto di almeno due gruppi, il cui numero dipende dai valori (detti anche livelli o condizioni) che la variabile indipendente può assumere. Di conseguenza, nella ricerca sull'esito abbiamo due gruppi (un gruppo sperimentale - sottoposto a trattamento - e un

\footnotetext{
${ }^{3}$ Quest'ultimo risultato è noto come "paradosso dell'equivalenza" (Stiles, Shapiro, \& Elliot, 1986), secondo il quale la psicoterapia produce i medesimi effetti, indipendentemente dai modelli teorico-clinici e dalle tecniche usate. Tale equivalenza degli esiti prodotti da diverse psicoterapie può essere considerata un paradosso alla luce della diversità "tecnica" che a) contraddistingue $i$ diversi orientamenti psicoterapentici, e che b) è supposta riflettersi in una specificità degli esiti da parte di psicoterapie di diverso orientamento.
} 
Ricerca in Psicoterapia / Research in Psychotherapy 2010; 1 (13): 61-91. http://www.researchinpsychotherapy.net gruppo di controllo ${ }^{4}$ - non sottoposto a trattamento); nel caso della ricerca comparata sull'esito - che intende confrontare l'efficacia di più trattamenti tra loro - abbiamo invece a che fare con almeno due gruppi sperimentali [(ciascuno dei quali sottoposto a un trattamento); per una dettagliata rassegna e discussione della ricerca sull'esito si veda Crits-Christoph (1997); Lambert \& Archer (2007); Wampold (2001)].

La differenza sostanziale tra i due approcei è determinata dal diverso grado di controllo che è possibile esercitare sulle variabili intervenienti che potrebbero minacciare la validità delle conclusioni tratte dai risultati dello studio. ${ }^{5}$ Tale controllo viene esercitato principalmente attraverso l'assegnazione randomizzata dei partecipanti (i pazienti) ai diversi livelli della variabile dipendente (il trattamento) e, di conseguenza, ai gruppi di pazienti Lad esempio: 0 = assenza di trattamento (gruppo di controllo); livello 1 = trattamento $_{1}$ (gruppo sperimentale $_{1}$ ); livello $2=$ trattamento $_{2}$ (gruppo sperimentale 2 ), etc.)l. Negli studi sperimentali (che nella ricerca clinica e psicoterapentica vengono anche chiamati Randomized Controlled Trials $^{6}$ - RCT) è possibile la randomizzazione, per cui è di conseguenza possibile "formare" tanti gruppi quanti sono i livelli della variabile indipendente. Ad esempio, è possibile assegnare casualmente i pazienti che si presentano in un ambulatorio psicoterapentico alle varie condizioni sperimentali, cioè stabilire casualmente chi farà una terapia (ed eventualmente quale) e chi no.

Al contrario, negli studi quasi-sperimentali (chiamati anche Preferential Trials) non è possibile o si preferisce non adoperare una procedura del genere. In questo caso, gli sperimentatori utilizzano gruppi "già esistenti", la cui formazione è avvenuta in base a criteri non controllati dal ricercatore (ad esempio, nello studiare la terapia psicoanalitica e cognitivo-comportamentale, sono il consiglio del medico di base, le inclinazioni personali del paziente, l'esito delle prime interviste, etc. a stabilire chi farà una terapia piuttosto che unaltra). In entrambi i casi, è necessario ridurre il più possibile la variabilità dei trattamenti somministrati. A tal fine, nel corso degli anni '90 si svilupperanno, all'interno del movimento degli Empirically Supported Treatments (EST; Task Force on Promotion and Dissemination of Psychological Procedures, 1995), i cosiddetti trattamenti "manualizzati" - e cioè standardizzati - in cui vengano resi espliciti e descritti quali principi e quali tecniche devono essere utilizzate dai terapeuti per il trattamento di quali disturbi (per un approfondimento si veda Migone, 1986, 1989). In questo ambito, specifiche procedure dovrebbero inoltre essere utilizzate per valutare il grado di aderenza tra la condotta effettiva dei terapenti e le linee guida esplicitate nei manuali. ${ }^{\gamma}$

\footnotetext{
${ }^{4}$ Nella ricerca in psicoterapia, il gruppo di controllo dovrebbe essere idealmente sottoposto agli effetti di una qualche attività il più possibile simile al trattamento psicoterapentico di cui si vuole studiare l'efficacia, ma che non contiene nessun "ingrediente" considerato essere attivo nel trattamento in esame - in analogia con la condizione placebo della ricerca medica. Una strategia tipicamente utilizzata per costituire un gruppo di controllo nella ricerca in psicoterapia è quella dell'impiego delle cosiddette "liste d’attesa": ai soggetti viene comunicato che saranno sottoposti a un trattamento psicoterapeutico, che però non avrà inizio fino alla fine dello studio. Per una discussione critica sull'uso dei gruppi di controllo nella ricerca sull'esito in psicoterapia si veda Borkovec \& Sibrava (2005).

${ }^{5}$ Per una rassegna generale sulla metodologia di ricerca e l'analisi dei dati in ambito clinico si veda Morgan (2006).

${ }^{6} \mathrm{Si}$ usa anche comunemente il termine Randomized Clinical Trials.

${ }^{7}$ La manualizzazione dei trattamenti e il loro impiego all'interno degli RCT rientrano all'interno della cosiddetta Evidence-Based Medicine, secondo la quale la valutazione della
} 
Ricerca in Psicoterapia / Research in Psychotherapy 2010; 1 (13): 61-91. http://www.researchinpsychotherapy.net

Lo studio dell'efficacia psicoterapentica comporta la necessità di studiare gli effetti di un trattamento al passare del tempo, e implica così lo studio di due variabili indipendenti: il "trattamento" (con ad esempio due livelli/condizioni: $\mathbf{0}=$ trattamento e $\mathbf{1}=$ assenza di trattamento) e il "cambiamento nel corso del tempo" (con ad esempio due livelli/condizioni: 1 = pre-trattamento e 2 = post-trattamento). ${ }^{8}$ In questo caso, vengono utilizzati i cosiddetti disegni di ricerca misti. ${ }^{9}$ A differenza dei disegni trasversali che rilevano e utilizzano il valore delle osservazioni in diversi gruppi - e dei disegni longitudinali - che rilevano e utilizzano il valore delle osservazioni in diversi momenti, cioè il loro ordine temporale - $\mathrm{i}$ disegni misti integrano questi due aspetti, cioè permettono di studiare gli effetti di una variabile indipendente su una o più variabili dipendenti al passare del tempo in due o più gruppi. I disegni misti che seguono un approccio sperimentale prendono anche il nome di "disegni pre-post test con gruppo di controllo" e corrispondono agli RCT; i disegni misti che seguono un approccio quasi sperimentale vengono chiamati "disegni pre-post test con gruppo di controllo non equivalente", e corrispondono ai Preferential Trials.

Questo tipo di disegni di ricerca richiede la misurazione della variabile dipendente (ad esempio, la sintomatologia) lungo due dimensioni: una dimensione tra i soggetti o trasversale (in relazione ai diversi gruppi che ricevono i diversi livelli della variabile indipendente "trattamento") e una dimensione entro i soggetti o longitudinale (in relazione ai diversi momenti in cui la misurazione viene effettuata, corrispondenti ai livelli della variabile "tempo"). I dati raccolti lungo queste due dimensioni vengono chiamati dati panel, e comportano losservazione degli effetti di una variabile dipendenti a) in successivi momenti temporali (ad esempio: pre- e post-trattamento) e in uno o più gruppi (ad esempio: gruppo sperimentale che riceve il trattamento e gruppo di controllo che non riceve il trattamento). ${ }^{10}$

pratica psicoterapentica deve essere ricondotta a criteri basati sull'evidenza (per una discussione si veda Del Corno e Lang, 2006).

${ }^{8}$ In inglese si utilizza il termine trial per riferirsi allo studio degli effetti di una variabile nel corso del tempo all'interno di un'ottica sperimentale o quasi sperimentale.

${ }^{9}$ Questo nome deriva dal fatto che questi studi, di fatto, integrano i disegni di ricerca tra $i$ soggetti ed entro i soggetti. Nei primi, detti anche trasversali, ogni soggetto riceve solo uno dei possibili livelli/condizioni di una variabile indipendente in un momento determinato. Questi disegni permettono di indagare la variabilità tra i soggetti. Nei secondi, detti anche longitudinali o a misure ripetute, ogni soggetto riceve invece tutti i livelli/condizioni di una variabile indipendente in diversi momenti. Questi disegni permettono di studiare la variabilità entro i soggetti. Affinché un disegno di ricerca sia definito misto è necessario che: a) si vogliano studiare gli effetti di due variabili indipendenti su almeno una variabile dipendente; b) la variabile dipendente venga misurata sia tra i soggetti che entro i soggetti; c) la variabile dipendente misurata entro i soggetti faccia riferimento agli effetti nel tempo della variabile indipendente.

${ }^{10}$ I dati raccolti relativamente a più variabili in un singolo momento temporale vengono chiamati dati trasversali (in cui solo il valore delle osservazioni assume rilevanza), mentre i dati raccolti relativamente a una o più variabili in successivi momenti temporali vengono chiamati dati longitudinali (o anche serie temporali, dove assume rilevanza sia il valore delle variabili sia l'ordine temporale con cui questi valori sono stati ottenuti). Quando le osservazioni relative a una o più variabili vengono condotte in modo da includere entrambi questo tipo di dati, si parla di dati panel (panel data). Questo tipo di dati è estremamente diffuso nella ricerca in psicoterapia. Si pensi, ad esempio alla ricerca sul processo, in cui determinate variabili (ad esempio, gli schemi relazionali) vengono misurate sia in diversi momenti (ad esempio, durante il trattamento) che in diversi gruppi (ad esempio, nel paziente e nel terapenta). 
Ricerca in Psicoterapia / Research in Psychotherapy 2010; 1 (13): 61-91. http://www.researchinpsychotherapy.net

Livelli di osservazione. ${ }^{11}$ La ricerca sull'esito misura l'efficacia in riferimento a diverse variabili dipendenti, che solitamente includono diversi aspetti della sintomatologia e della personalità del paziente così come la qualità delle sue relazioni interpersonali o il suo grado di funzionamento. In queste prime fasi della ricerca sull'esito, tali variabili vengono rilevate all'inizio del trattamento (pre-treatment), al termine di esso (post-treatment), ed eventualmente in un periodo successivo al termine del trattamento (follow up) [di conseguenza, nella tradizionale ricerca sull'efficacia, l'esito viene inteso esclusivamente nei termini del miglioramento clinico del paziente osservabile al termine del trattamento detto anche esito finale ${ }^{12}$ o "big- $O{ }^{\prime}$; Greenberg (1986)]. ${ }^{13}$

I primi strumenti utilizzati a tal fine sono questionari autosomministrati. Tra questi ricordiamo lo Shapiro Personal Questionnaire (1961) e i più noti Beck Depression Inventory (BDI; Beck, Ward, Mendelson, Mock, \& Erbaugh, 1961); per la versione più recente si veda Beck, Steer, \& Brown, 1996), e la Symptom Check List 90-Revised (SCL90-R; Derogatis, 1977; Derogatis, 1992). A questi seguiranno, in tempi recenti, l'Inventory of Interpersonal Problems (IIP; Horowitz, Rosenberg, Baer, Ureño, \& Villaseñor, 1988), il Clinical Outcome Routine EvalutaionOutcome Measure (CORE-OM; Evans, et al., 2000) e l'Outcome Questionnaire-45 (OQ-45; Lambert et al., 2004). Esistono inoltre anche scale di misurazione che possono essere somministrate da terapenti addestrati (ad esempio: l'Hamilton Rating Scale for Depression - HRSD; Hedlung \& Vieweg, 1979) o da altri significativi Lad esempio: il Family Life Questionnaire - FLQ; Guerney (1978); per una rassegna e approfondimento si veda Hill \& Lambert, 2004 e Zennaro, Cristofanelli, \& Vottero Ris, (2006)].

Analisi dei dati. L'analisi dei dati così raccolti avviene attraverso procedure di elaborazione statistica che consentono di verificare se esiste una differenza significativa tra $\mathrm{i}$ valori medi delle variabili di esito misurate rispettivamente nel corso del tempo (valori pre, post e follow up) e tra i gruppi (valori del gruppo sperimentale e del gruppo di controllo). L'obiettivo è dunque quello di confrontare due o più valori di due o più variabili in diversi gruppi al passare del tempo. Una delle strategie di analisi maggiormente utilizzate è l'analisi della varianza (ANOVA) per misure ripetute, che permette di valutare se esiste un'interazione significativa tra i cambiamenti della variabile indipendente (ad esempio, la sintomatologia) misurata entro i soggetti (valori pre-post) e tra i soggetti (gruppo sperimentale e di controllo). In altri termini, è possibile valutare contemporaneamente: a) se i due gruppi, nel loro insieme, cambiano nel corso del tempo; b) se tra i due gruppi ci sono differenze, indipendentemente dal passare del tempo; c) se ci sono differenze tra i gruppi nel corso del tempo. Nel caso di più di due rilevazioni temporali (ad esempio: pre, post e follow up) è necessario - dopo aver verificato la significativa interazione tra

\footnotetext{
11 È opportuno distinguere i livelli di osservazione, con cui in questo articolo facciamo riferimento alla scala temporale in relazione alla quale avviene la raccolta dei dati (ad esempio: prima e/o dopo il trattamento, dopo le sedute, o durante le sedute) e i livelli di misurazione, con cui si fa invece riferimento al tipo di dati che vengono raccolti (nominali, ordinali, a intervalli o a rapporti).

${ }^{12}$ Greenberg (1986) propone una distinzione tra esito finale (osservato al termine del trattamento), intermedio (osservato durante il trattamento alla fine delle sedute), e immediato (osservato durante le sedute).

${ }^{13}$ Tuttavia, col passare del tempo, si assisterà a una sempre più frequente misurazione dell'esito in un periodo intermedio del trattamento (mid-treatment).
} 
Ricerca in Psicoterapia / Research in Psychotherapy 2010; 1 (13): 61-91. http://www.researchinpsychotherapy.net

la variabile entro i soggetti e quella tra i soggetti attraverso l'ANOVA applicare un $t$-test per campioni appaiati al fine di stabilire con esattezza quando sia avvenuto il cambiamento (cioè tra quali misurazioni: $t_{0}-t_{1}, t_{1}-t_{2}$, $t_{0}-t_{2}$, etc.). ${ }^{14}$

Inoltre, come già osservato, la meta-analisi può essere impiegata quando si vogliono valutare e riassumere i risultati di una serie di studi presenti in letteratura. L'analisi statistica all'interno delle meta-analisi viene condotta attraverso il calcolo dell'effect size (entità dell'effetto), che permette prima di valutare l'entità della modificazione della variabile dipendente (ad esempio, sintomatologia) in due gruppi (ad esempio, trattato e non trattato) in ciascuno studio considerato, e poi di ottenere un valore medio (relativamente al numero totale di studi considerati) che rappresenta un indice dell'entità media degli effetti presenti nei trattamenti considerati.

\section{La ricerca sul processo}

Il processo terapeutico viene tradizionalmente definito come quell'insieme di fenomeni, avvenimenti e/o attività ascrivibili al paziente, al terapenta e alla loro relazione, osservabili nel corso di una psicoterapia (Kiesler, 1973; per una definizione più recente ma essenzialmente analoga si veda Orlinsky, Rønnestad, \& Willutzki, 2004). Lo studio del processo consiste, dunque: a) nel selezionare un livello d'analisi che permetta di osservare ciò che accade durante il trattamento; b) nell’identificare quali variabili siano più adeguate per descrivere $\mathrm{i}$ fenomeni oggetto d'interesse al livello d'analisi selezionato; c) nel descrivere la frequenza o la percentuale di occorrenza con cui questi fenomeni si presentano; d) nel valutare la relazione tra i costrutti selezionati cosi come l'eventuale diversa distribuzione di questi fenomeni tra diversi gruppi o nel tempo.

Una prima forma di ricerca sul processo è rintracciabile già nel lavoro di Freud che, attraverso quello che oggi potremmo definire una forma poco sistematica di studio narrativo del caso, descrive il trattamento del paziente sulla base degli appunti presi durante le sedute e l'utilizzo della propria memoria relativamente agli episodi salienti della seduta (si veda ad esempio il caso de L'uomo dei topi; Freud, 1909). In questo periodo, inoltre, si osservano i primi tentativi di studiare aspetti del processo psicoanalitico attraverso il metodo sperimentale. Un esempio è il lavoro di Meltzer (1930), che cercò di indagare il costrutto della repressione attraverso lo studio del rapporto tra memoria ed emozioni (positive vs negative).

Tuttavia, la nascita della moderna ricerca sul processo terapeutico può essere fatta risalire al lavoro di Carl Rogers (1942), che fu il primo a utilizzare in modo sistematico i trascritti delle sedute per studiare ciò che accadeva durante le sedute. ${ }^{15}$ Lo studio del processo è in questa fase caratterizzato dall'applicazione di specifici strumenti per misurare in modo "obiettivo" e attendibile eventi quantificabili all'interno delle registrazioni delle sedute. I costrutti che vengono maggiormente indagati in questa fase riguardano la relazione terapeutica e fanno principalmente riferimento all'approceio centrato sul cliente di Rogers (1951, 1957, 1975). Tuttavia, si assiste anche allo sviluppo di strumenti per misurare costrutti di matrice

\footnotetext{
${ }^{14}$ Nel caso le variabili in esame non presentino una distribuzione normale, possono essere usate delle statistiche non parametriche quali ad esempio il test di Kruskal-Wallis, il test di Mann-Whitney, il test di Wilcoxon o il test esatto di Fisher).

${ }_{15}$ L'inizio dell'utilizzo dei trascritti delle sedute psicoterapeutiche risale al 1929 (Dittes, 1959).
} 
Ricerca in Psicoterapia / Research in Psychotherapy 2010; 1 (13): 61-91. http://www.researchinpsychotherapy.net psicoanalitica quali la profondità dell'interpretazione (Harway, Dittman, Raush, Bordin, \& Rigler, 1955), il transfert (Fielder, 1951) e i meccanismi di difesa (Glesser \& Ihilevic, 1969; Milbrath et al., 1983). Contemporaneamente, si sviluppa l'analisi del contenuto dei trascritti, per cui vengono creati numerosi sistemi di codifica del comportamento verbale di paziente e/o terapenta [per una rassegna dei risultati ottenuti con tali strumenti in questa prima fase della ricerca sul processo si veda Marsden, (19\%1)]. Questi sistemi di codifica del contenuto si evolveranno successivamente nei cosiddetti Verbal Response Modes (VRM), che intendono categorizzare il comportamento verbale di paziente e terapeuta (Stiles, 1978, 1979) o del solo terapenta (Hill, 1978; Friedlander, 1982) nel corso delle sedute. Infine, si assiste allo sviluppo di una serie di strumenti atti a misurare la struttura (Benjamin, 1974) e la qualità (Luborsky, 1976, 1983; Hartley \& Strupp, 1983) delle relazione terapentica secondo una prospettiva trans-teoretica Lper una rassegna degli strumenti maggiormente utilizzati in questa prima fase della ricerca sul processo si veda Kiesler, (1973)].

Approccio e disegni di ricerca. La ricerca sul processo è caratterizzata da un approccio non sperimentale anche se, nel corso di questa fase iniziale della ricerca sul processo, si osserva il tentativo di applicare l'approccio sperimentale allo studio del caso singolo (si veda ad esempio Leitenberg, Wincze, Butz, \& Callahan, 1970). A differenza dell'approceio sperimentale e quasi sperimentale, l'approcio non sperimentale non analizza la relazione tra due o più variabili in termini di rapporto di cansa-effetto, bensì nei termini di associazioni o covariazioni. Ciò è dovuto al fatto che l'ipotetica variabile indipendente (che in questo caso corrisponde a un aspetto del processo terapeutico) non viene manipolata - per impossibilità o per scelta ma semplicemente osservata. In altri termini, il ricercatore studia le caratteristiche o qualità di un gruppo o di un individuo che rappresentano dei loro attributi specifici. ${ }^{16}$ L'obiettivo non è dunque quello di valutare se una o più variabili indipendenti (il fatto di essere sottoposti a un trattamento) determinano degli effetti in una o più variabili dipendenti (ad esempio, la sintomatologia), bensì quello di individuare correlazioni e/o fare previsioni relativamente al rapporto tra due o più variabili (che misurano determinati aspetti del processo). Per questo motivo tale approccio viene anche definito correlazionale. ${ }^{17}$

\footnotetext{
${ }^{16}$ Mentre la ricerca sperimentale presenta una maggior validità interna (dovuta al maggior controllo di quei fattori che potrebbero minacciare le inferenze relative agli effettivi rapporti tra le variabili studiate) ed esterna (dovuta al maggior controllo sulle procedure di campionamento che permettono di generalizzare i risultati ottenuti dal campione studiato alla popolazione di riferimento), la ricerca non sperimentale presenta una maggior validità ecologica (che indica il grado in cui i comportamenti studiati nel corso della ricerca riflettono quelli che si osserverebbero effettivamente in contesti di vita quotidiana).

${ }^{1} \gamma$ Un altro termine utilizzato per descrivere questo approceio è quello di passivoosservazionale (Kazdin, 1994), in riferimento al fatto che la variabili indipendenti non vengono manipolate come negli approcei (quasi) sperimentali. Gli studi condotti secondo un approccio correlazionale vengono anche definiti naturalistici, in riferimento al fatto che la rilevazione delle variabili avviene in setting naturali, cioè in cui il ricercatore può esercitare un controllo estremamente ridotto. Il termine "correlazionale" viene a volte criticato per il fatto di riferirsi a una specifica modalità di analisi dei dati (l’indice di correlazione) che, benché molto usata in questo approccio, non rappresenta l'unico modo in cui i dati possono essere analizzati - è ad esempio possibile confrontare medie, frequenze, etc.). Nel corso del presente articolo, tuttavia, faremo comunque riferimento a tale termine per il suo ormai diffuso utilizzo all'interno della letteratura scientifica internazionale sulla ricerca in psicoterapia.
} 
Ricerca in Psicoterapia / Research in Psychotherapy 2010; 1 (13): 61-91. http://www.researchinpsychotherapy.net

Esistono tuttavia una serie di procedure di analisi statistica in cui la misura del rapporto tra due o più variabili può permettere di fare inferenze relative a possibili rapporti di causa-effetto tra esse. È il caso dei modelli statistici essenzialmente basati sull'analisi della regressione [ad esempio, l'analisi della regressione semplice e multipla, la path analysis e i modelli di equazioni strutturali; per un approfondimento si veda Russell (1995)]. ${ }^{18}$

La ricerca sul processo di questo periodo fa uso di disegni di ricerca prevalentemente trasversali e longitudinali. Nel primo caso, aspetti specifici del processo terapentico vengono misurati all'interno e/o dopo le sedute considerate e poi messi a confronto in riferimento a diversi gruppi. Ad esempio, è possibile confrontare il comportamento verbale di terapeuti appartenenti a diverse scuole (ad esempio, Hill, Thames, \& Rardin, 1979), oppure valutare, in relazione a una specifica seduta, i livelli di accordo tra le intenzioni dei terapenti e le reazioni dei pazienti (Hill, Thomson, Cogar \& Denman, 1993). Ciò che rende questi disegni trasversali consiste nel fatto che, benché le variabili di processo possano essere misurate anche in diversi momenti della terapia nei diversi gruppi, il confronto viene fatto tra i valori medi aggregati all'interno di ciascun gruppo. Il focus è dunque sulla variabilità tra i soggetti.

Nel caso di disegni longitudinali, al contrario, specifici aspetti del processo terapeutico vengono misurati nel corso delle sedute e/o dei trattamenti, con l'obiettivo di studiare il rapporto tra le variabili oggetto di interesse al passare del tempo. Ad esempio, è possibile studiare e confrontare la diversa distribuzione del comportamento di un gruppo omogeneo di pazienti a inizio, metà e fine terapia. A tal proposito, è opportuno osservare che lo studio del caso singolo $(\mathrm{N}-1)$ che, nel corso degli anni verrà notevolmente rivalutato nella ricerca in psicoterapia, rappresenta una specifica declinazione del disegno di ricerca longitudinale (si veda Elliott, 2002; Hilliard, 1993; Lingiardi, 2006).

Infine, anche se raramente, la ricerca di processo di questo periodo fa uso di disegni di ricerca misti, che permettono ad esempio di analizzare se il comportamento verbale di pazienti e terapeuti (variabile tra $i$ soggetti) viene utilizzato in maniera diversa all'interno di diverse fasi del trattamento (variabile entro i soggetti).

Livelli di osservazione. La ricerca sul processo è caratterizzata sin dai suoi esordi da una misurazione delle variabili nel corso delle sedute (osservazioni within-session). Ciò avviene attraverso l'applicazione di specifici strumenti di analisi dei trascritti terapeutici. Tuttavia, è opportuno osservare che, nel corso di questa prima fase, si farà sempre più spesso uso di specifici questionari - compilati da paziente e/o terapeuta - che permettono di misurare aspetti del processo alla fïne di una o più sedute. Ciò è dovuto alla riconosciuta esigenza di studiare ciò che accade durante il processo terapentico facendo riferimento anche all'esperienza soggettiva di paziente e/o terapeuta (Orlinsky \& Howard, 1986). Si introduce così la distinzione tra sistemi di codifica osservazionali e self-report [per una rassegna attuale si veda Freni \& Papini (2006); Greenberg \& Pinsof (1986); Lepper \& Riding (2006)]. I primi implicano lo studio dei trascritti ${ }^{19}$ delle sedute da parte di terzi (che a volte possono comunque coincidere con il terapenta) e

\footnotetext{
${ }^{18}$ Questo tipo di analisi statistiche permette di esaminare rapporti di causazione tra una o più variabili indipendenti (dette anche predittori) e una o più variabili dipendenti (dette anche variabili spiegate) nella misura in cui è possibile valutare se al variare delle prime si produce un effetto sulle seconde (detto anche effetto di produzione).

${ }^{19} \mathrm{Nel}$ corso degli anni si farà sempre più frequente l'utilizzo delle registrazioni video delle sedute.
} 
Ricerca in Psicoterapia / Research in Psychotherapy 2010; 1 (13): 61-91. http://www.researchinpsychotherapy.net

permettono così di misurare determinati aspetti del processo terapentico nel corso delle sedute (osservazioni within-session). Esempi di questo tipo sono le scale sviluppate da Truax e Carkhuff (196\%) per misurare aspetti quali l'empatia (Truax Accurate Empathy Scale - TAES), l'attenzione positiva incondizionata (Truax Unconditional Positive Regard Scale - TUPRS) e la genuinità (Truax Genuineness Scale - TGS); l'Experiencing Scale (Klein, Mathieu, Gendlin, and Kiesler, 1969), che permette di analizzare nei trascritti i livelli di elaborazione e coinvolgimento affettivo del paziente nel corso delle sedute; la Depth of Interpretation Scale, sviluppata per misurare i livelli di interpretazione del terapeuta (Harway et al., 1955); i Verbal Response Modes (VRM; si veda ad esempio Freidlander, 1982; Hill, 1978; Stiles, 1978, 1979), che permettono di valutare il comportamento verbale di paziente e/o terapeuta nel corso delle sedute; infine, una serie di strumenti per misurare l'alleanza terapentica, quali le Penn Helping Alliance Scales (PHAS; Luborsky, 1976, 1983) e la Vanderbilt Therapeutic Alliance Scale (VTAS; Hartley \& Strupp, 1983). Al contrario, i self-report sono dei questionari auto-somministrati che vengono compilati da paziente e/o terapenta al termine delle sedute (osservazioni post-session), permettendo così di misurare aspetti diversi del processo terapentico dalla prospettiva soggettiva dei contraenti la relazione terapentica. ${ }^{20}$ Esempi di questionari autosomministrati sono il Defense Mechanisms Inventory (DMI; Glesser \& Thilevic, 1969) e il Defense Style Questionnaire (DSQ; Bond, 1986), che si propongono di indagare le difese del paziente da una prospettiva psicoanalitica, e il Barrett-Lennard Relationship Inventory (BarretLennard, 1962) che intende misurare le "condizioni facilitanti" il cambiamento terapentico secondo l’approccio di Rogers (195\%). Infine, da lì a poco, si svilupperanno i cosiddetti session reports (Orlinsky \& Howard, 1986) che consentono di indagare svariati aspetti del processo terapentico attraverso la prospettiva sia del paziente che del terapenta.

E importante qui osservare che la maggior parte degli studi di questo periodo fanno uso di una selezione ad hoc del "quando" osservare o rilevare le variabili di interesse. Ciò avviene attraverso una procedura definita di campionamento temporale, sulla base della quale le variabili di interesse vengono osservate, misurate, e di conseguenza studiate, solo in determinati momenti della seduta (ad esempio, i primi 5 minuti della parte iniziale, intermedia e finale della seduta a loro volta selezionate, ad esempio, dalla parte iniziale, intermedia e finale della terapia). Anche in questo caso, tuttavia, dalla metà degli anni ' $\%$, sarà possibile riscontrare una misurazione sempre più frequente nel corso delle sedute e/o dei trattamenti.

Analisi dei dati. I dati così ottenuti, una volta rappresentati in forma di percentuali di occorrenza, frequenze e/o medie, vengono analizzati attraverso procedure di analisi statistica (ad esempio, indici di correlazione, chi-quadro, $t$-test per campioni indipendenti o appaiati, ANOVA per campioni indipendenti o per misure ripetute, analisi della regressione). Le

\footnotetext{
20 Altri autori (ad esempio, Orlinsky \& Howard, 1978; Greenberg \& Pinsof, 1986) - per distinguere gli strumenti di misurazione in base alla prospettiva di osservazione - parlano piuttosto di sistemi di osservazione rispettivamente non partecipante e partecipante. Benché spesso ci sia corrispondenza tra le due diciture (e cioè tra strumenti osservazionali e sistemi di osservazione non partecipante da una parte, e tra self-report e sistemi d'osservazione partecipante dall'altra), ciò non sempre vero. $\mathbf{E}$ il caso di quando uno strumento osservazionale per l'analisi dei trascritti viene utilizzato dal terapenta stesso che ha condotto la seduta oggetto di studio. In questo caso, lo strumento viene utilizzato dalla prospettiva di uno dei due partecipanti all'incontro terapeutico.
} 
Ricerca in Psicoterapia / Research in Psychotherapy 2010; 1 (13): 61-91. http://www.researchinpsychotherapy.net

analisi vengono condotte utilizzando dati aggregati. ${ }^{21}$ Nei disegni di ricerca trasversali, i dati vengono solitamente aggregati in riferimento ai gruppi che si vogliono confrontare, come ad esempio il comportamento a) di pazienti vs terapenti, b) di un certo tipo di pazienti con un altro tipo di pazienti, e/o c) di un certo tipo di terapenti con un altro tipo di terapenti. Nei disegni longitudinali, l'aggregazione dei dati viene fatta con riferimento ai diversi momenti che si vogliono confrontare, come ad esempio a) le fasi della seduta (parte iniziale, intermedia e finale), ${ }^{22}$ b) ciascuna seduta, e/o c) le fasi della terapia (parte iniziale, intermedia e finale). Infine, nei disegni misti, l'aggregazione dei dati viene fatta in riferimento sia ai gruppi che ai momenti temporali.

\section{Considerazioni teorico-metodologiche e relative all'analisi dei dati}

\subsection{Esito terapeutico}

a) L'esito terapentico viene considerato un fenomeno di natura lineare, cioè che si modifica proporzionalmente al passare del tempo. ${ }^{23}$ In altri termini, l'esito terapeutico è considerato modificarsi (in maniera crescente o decrescente) a una velocità costante. Ciò giustifica la convinzione che, per valutare l'esito di un trattamento, sia sufficiente misurare determinate variabili di esito (sintomatologia, problemi interpersonali, etc.) all'inizio $\left(t_{0}\right)$ e alla fine $\left(t_{1}\right)$ del trattamento ed, eventualmente, in un periodo di follow up $\left(t_{2}\right)$.

In realtà, l'esito terapeutico è un processo che si modifica al passare del tempo con una velocità che può anche non essere costante; di conseguenza, le variazioni che l'esito può presentare lungo tutto il corso del trattamento non dovrebbero essere trascurate. Per studiare il cambiamento non è sufficiente determinarne l'entitá, ma è necessario studiarne la forma. Si consideri l'esempio per cui la sintomatologia depressiva di un gruppo di pazienti, misurata all'inizio, a metà, e alla fine del trattamento, evidenzia una progressiva riduzione che indica un miglioramento clinico (Figura 1). Questa informazione, benché rilevante nella misura in cui ci fornisce informazioni sulle variazioni di depressività in termini assoluti tra l'inizio, la metà e la fine della terapia, nulla ci dice sui livelli di questa variabile durante la terapia. La riduzione nei livelli di depressione osservati tra l'inizio e la fine del trattamento viene in questi casi interpretata come una riduzione lineare (Figura 1, andamento A). Tuttavia, è possibile che i livelli di depressione siano aumentati subito dopo la prima seduta per poi ridursi notevolmente nei pressi della seduta intermedia e, dopo di essa, essersi ridotti per poi aumentare nuovamente nei pressi della seduta finale (Figura 1, andamento B). In assenza di un assessment più frequente lungo il corso del trattamento, risulta impossibile valutare se quello che noi supponiamo

\footnotetext{
${ }^{21}$ Il processo di raccolta di dati implica la rilevazione di determinate variabili in singoli soggetti (disegni trasversali), in singoli momenti (disegni longitudinali) o in entrambi (disegni misti). L'analisi di dati aggregati consiste nell'analizzare il rapporto tra due o più variabili facendo riferimento non ai dati raccolti nei singoli soggetti e/o momenti, bensì aggregando le singole rilevazioni con riferimento ai gruppi e/o ai momenti che si vogliono confrontare.

${ }_{22}$ È chiaro come, in relazione a quanto detto, l'aggregazione con riferimento a parti della seduta è possibile solo se i dati sono stati raccolti attraverso l'analisi dei trascritti.

${ }^{23}$ Da un punto di vista matematico, un cambiamento lineare può essere descritto da un polinomio di $1^{\circ}$ grado della forma $y=m x+b$. In un'equazione di questo tipo, $b$ rappresenta l'intercetta - cioè il valore iniziale (in $t_{0}$ ), mentre $m$ rappresenta la pendenza - cioè la velocità con cui $y$ si modifica al passare del tempo.
} 
Ricerca in Psicoterapia / Research in Psychotherapy 2010; 1 (13): 61-91. http://www.researchinpsychotherapy.net essere una riduzione lineare sia invece effettivamente un andamento nonlineare che, tuttavia, interseca i valori rilevati nelle tre misurazioni. A tal proposito è utile osservare che, nel corso degli anni che caratterizzeranno le fasi successive della ricerca sull'esito, emergerà la consapevolezza dell'opportunità di "monitorare" gli andamenti dell'esito lungo tutto il corso del trattamento. Ciò si risolverà in una tendenza a misurare l'esito sempre più frequentemente anche nel corso del trattamento (esito intermedio; Greenberg, 1986), attraverso la somministrazione al termine delle sedute di alcuni degli strumenti descritti sopra.

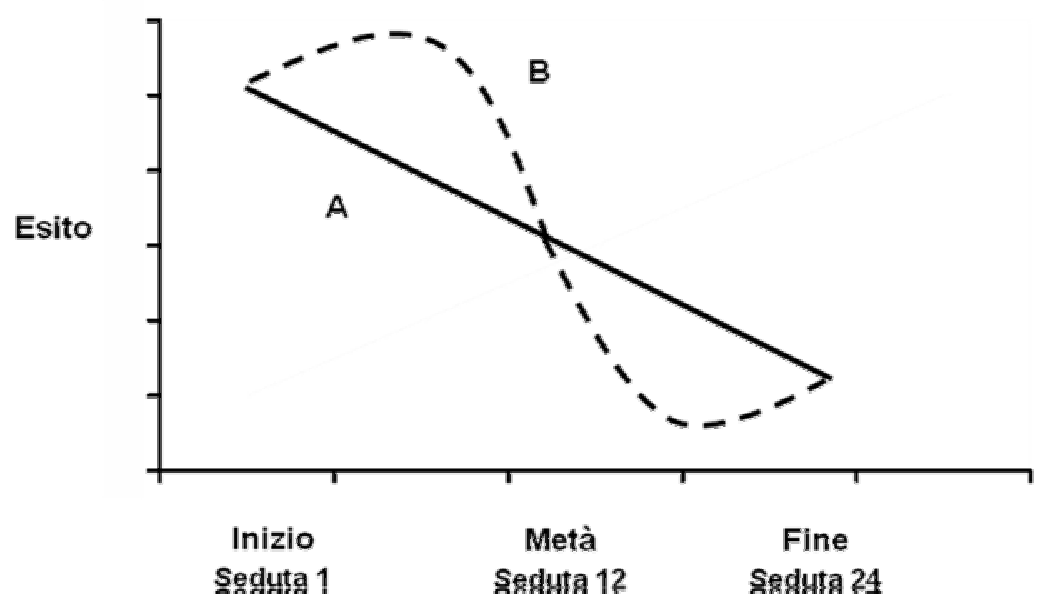

Figura 1. Due diversi andamenti della sintomatologia (A: lineare e B: non lineare) che possono intersecare $i$ medesimi valori nel corso di tre misurazioni.

b) L'esito terapentico non viene considerato nella sua natura processuale, bensi viene inteso come un fenomeno di tipo incrementale [per una discussione si veda Francis, Fletcher, Stuebing, Davidson, \& Thomson (1991)]. Ciò significa che il cambiamento viene concepito come l'incremento tra $i$ valori di una variabile $y$ in $t_{0}$ e $t_{1}$, e viene analizzato utilizzando dati aggregati nei termini della differenza di valori medi Diff $=x_{\text {pre }}-x_{\text {post }}$ (difference score). Tale approccio è problematico perché: 1) assume che il cambiamento sia un fenomeno lineare, per cui due misurazioni sono sufficienti per descrivere il cambiamento (vedi sopra); 2) il difference score è attendibile per misurare il cambiamento nella misura in cui i soggetti misurati al passare del tempo cambiano nello stesso modo (alla medesima velocità). Mentre il primo di questi due assunti è discutibile per la ovvia non linearità del cambiamento, il secondo assunto denuncia uno degli aspetti di quello che Kiesler $(1966,1983)$ definisce mito dell'uniformita, in base al quale i pazienti sono considerati cambiare allo stesso modo nel corso dei trattamenti.

c) I disegni di ricerca utilizzati rischiano di "appiattire" le differenze individuali. Il disegno di ricerca sperimentale utilizzato dalla ricerca sull'esito è di tipo estensivo, ${ }^{24} \mathrm{e}$ ha il merito di garantire la validità interna

\footnotetext{
${ }^{24}$ La distinzione tra ricerca estensiva e intensiva rispecchia, in gran parte, la distinzione tra approccio nomotetico (teso alla spiegazione di fenomeni attraverso la formulazioni di leggi o modelli di tipo generale) e approccio idiografico (teso alla comprensione il significato di fenomeni singoli, individuali ed, eventualmente, casuali). Nel primo, i
} 
Ricerca in Psicoterapia / Research in Psychotherapy 2010; 1 (13): 61-91. http://www.researchinpsychotherapy.net

dei risultati e la loro generalizzabilità alla popolazione da cui il gruppo sperimentale ̀̀ tratto - a condizione, ovviamente, che il campione sperimentale sia rappresentativo. Tuttavia, è importante sottolineare che $\mathrm{i}$ dati analizzati all'interno di un disegno di ricerca del genere rappresentano la media di una "performance" del gruppo di soggetti studiati (cioè, sottoposti a psicoterapia) e non la performance di un dato soggetto. Dunque, il fatto che i soggetti studiati presenti mediamente dei miglioramenti significativi in seguito a un trattamento non è garanzia del fatto che ciascun soggetto del gruppo abbia effettivamente tratto giovamento da esso. In altri termini, un disegno estensivo di tipo sperimentale può effettivamente dimostrare che un trattamento è efficace per un soggetto medio, ma non fornisce alcuna informazione sull'efficacia che quella terapia potrebbe avere per uno specifico individuo [per una discussione sulla necessità di riportare l’individuo al centro della ricerca in psicoterapia e più generalmente dell’indagine sui processi psicologici si veda Del Rio \& Molina (2008); Molenaar (2004); Molenaar \& Valsiner (2008); Salvatore \& Valsiner (2008)]. In tal senso, questo approccio allo studio dell'esito aderisce ad altro degli aspetti del mito dell'uniformità denunciato da Kiesler $(1966,1983)$, in base al quale la psicoterapia rappresenta un "pacchetto" standardizzato le cui proprietà non si modificano in base alle caratteristiche dei diversi pazienti e/o terapeuti, e tutti i pazienti possono essere considerati nguali e beneficiano allo stesso modo di un trattamento.

d) Viene proposto un modello medico di psicoterapia. Secondo il modello medico di psicoterapia, questa viene più o meno esplicitamente concepita come un trattamento caratterizzato da "ingredienti" specifici che 1) vengono realizzati attraverso specifiche tecniche terapentiche; 2) sono considerati avere un effetto altrettanto specifico sui problemi e/o disturbi presentati dal paziente. Ciò si riflette nell'approceio tipico della ricerca sull'esito descritto in questo articolo, in cui un trattamento (considerato essere caratterizzato da determinati ingredienti attivi) viene confrontato con un non-trattamento (in cui sono cioè assenti gli ingredienti attivi considerati responsabili degli effetti del trattamento in esame) o con un altro trattamento (caratterizzato cioè dalla presenza di altri ingredienti attivi). Tale modello acquisterà notevole rilevanza sfociando, negli anni '90, nell'implementazione di trattamenti manualizzati - che dovrebbero essere garanzia che il trattamento studiato "veicoli" gli ingredienti attivi supposti caratterizzare quel trattamento - all'interno del movimento EST (Task Force on Promotion and Dissemination of Psychological Procedures, 1995).

Nel corso degli anni, tuttavia, si assisterà allo sviluppo di un movimento "complementare" teso a mettere in discussione il modello medico della psicoterapia, che proporrà quello che viene definito modello contestuale

fenomeni oggetto di indagine vengono studiati prediligendo l'ampiezza delle osservazioni condotte quindi su un campione che deve essere necessariamente numeroso al fine di essere rappresentativo della popolazione di riferimento, ma in cui i soggetti vengono studiati in modo più "superficiale". Nel secondo caso, si predilige la profondita - ottenuta ad esempio utilizzando campioni meno numerosi ma lasciando più "spazio" ai soggetti studiati di esprimere i propri vissuti ed esperienze. Questi due approcci, solitamente considerati come due categorie distinte, rappresentano in realtà gli estremi di un continum su cui uno specifico disegno di ricerca si può variamente collocare in base a numerosi fattori. Senza entrare nel dettaglio [ per un approfondimento e discussione si veda Gelo, Braakmann, \& Benetka (2008)] è opportuno qui ricordare che i disegni di ricerca e le strategie di analisi tipiche della tradizionale ricerca in psicoterapia utilizzano un approccio che è esclusivamente estensivo (a scapito dell'aspetto intensivo) o intensivo (a scapito dell'aspetto estensivo). Tuttavia, recentemente si sono sviluppati nuovi approcei che permettono di integrare queste due dimensioni. 
Ricerca in Psicoterapia / Research in Psychotherapy 2010; 1 (13): 61-91. http://www.researchinpsychotherapy.net

della psicoterapia. Sulla base di questo, la psicoterapia è considerata essere efficace principalmente - anche se non esclusivamente - a causa dei suoi aspetti contestuali, cioè tutti quei fattori che non vengono esplicitamente descritti da uno o più modelli terapentici come elementi tecnici specifici e tipici di quel trattamento. Il movimento dell’integrazione in psicoterapia (Norcross \& Goldfried, 2005) così come il lavoro sulle Empirically Supported Relationships (Norcross, 2002) rappresentano dei movimenti coerenti con un modello contestuale della psicoterapia (per una discussione si veda Del Corno \& Lang, 2006).

e) Si fa uso di un approccio esclusivamente quantitativo. In accordo con il paradigma scientifico di matrice neo-positivistica che domina la ricerca in psicoterapia dagli anni '50 fino ai primi anni '80, lo studio dell'esito viene condotto all'interno di un approccio esclusivamente quantitativo. Ciò implica l'utilizzo di procedure per cui i dati vengono raccolti attraverso scale di misurazione e/o questionari e poi analizzati attraverso statistiche. A partire dagli anni '90, tuttavia, si svilupperà una riflessione critica sulla necessità di utilizzare (anche) approcei di tipo qualitativo nella ricerca in psicoterapia. All'interno dell'approccio qualitativo - che da una prospettiva epistemologica fa riferimento alla fenomenologia, all'ermenentica e all’interazionismo simbolico (Corbetta, 2009; Gelo, Braakmann, \& Benetka, 2008) - i dati vengono solitamente raccolti attraverso interviste aperte o semi-strutturate, e analizzati attraverso specifiche procedure di analisi qualitativa del testo (Bernard, 2009). Con particolare riferimento alla ricerca sull'esito, questo approccio permette di indagare il vissuto e il significato soggettivo di uno o più pazienti in riferimento all'impatto che la terapia ha avuto su di essi [Kuhnlein (1999); per un esempio di approccio integrato - quantitativo e qualitativo - allo studio dell'esito si veda anche Klein \& Elliott (2006)].

\subsection{Processo terapeutico}

a) II processo terapentico viene considerato un fenomeno omogeneo. In altri termini, il processo terapeutico è considerato presentare minime variazioni nel corso delle sedute e del trattamento - di natura lineare e continua. Tale assunto, che rappresenta un altro aspetto del mito dell'uniformità nella ricerca in psicoterapia denunciato da Kiesler (1966, 1983), rivela la propria infondatezza innanzitutto da una prospettiva clinica. Nel corso di una psicoterapia, infatti, ci troviamo ad esempio spesso di fronte a improvvise o inattese variazioni nel livello di introspezione del paziente, nel suo modo di riportare la propria esperienza emotiva o nella sua abilità a riflettere su di essa. Tali variazioni non devono essere "appiattite" dall'utilizzo di valori medi, ma devono essere adeguatamente misurate nel corso dell'intero trattamento. Inoltre, da un punto di vista metodologico, tale assunto sostiene implicitamente la convinzione che le variabili di processo misurate avrebbero un significato (cioè una funzione psicologica e/o relazionale all'interno della psicoterapia che viene studiata) costante nel tempo e indipendente dal contesto.

b) Si impiegano procedure di analisi statistica che fanno uso di valori medi aggregati tra le osservazioni compinte. Ciò determina una serie di conseguenze negative fondamentali: 
Ricerca in Psicoterapia / Research in Psychotherapy 2010; 1 (13): 61-91. http://www.researchinpsychotherapy.net

1. si corre il rischio di sovrastimare o sottostimare l'entita dei comportamenti osservati elo della loro relazione. Ad esempio, l'utilizzo di valori medi può impedire l'osservazione di valori eventualmente estremi, mentre la pratica psicoterapeutica, al contrario, ci insegna che variazioni "atipiche" nel comportamento del paziente possono essere particolarmente significative da un punto di vista clinico.

Inoltre, dato che il valore medio di una variabile è molto sensibile a suoi punteggi eventuali estremi, si corre il rischio di considerare come adeguati indicatori di una o più variabili valori che in realtà non lo sono. Infine, con riferimento ad esempio agli indici di correlazione, è possibile che una correlazione positiva (o negativa) tra i valori aggregati (tra i gruppi o entro i soggetti) di due determinate variabili possa oscurare una serie di correlazioni negative (o positive) che le due variabili potrebbero presentare all'interno di ciascun gruppo o di ciascun soggetto. Da un punto di vista matematico, infatti, i valori che producono una serie di correlazioni negative (tutte, idealmente, di $r=$ -1.00) nel corso del tempo o in diversi gruppi possono, se aggregate, produrre una correlazione di $r=+\mathbf{1 . 0 0}$ (Figura 2).

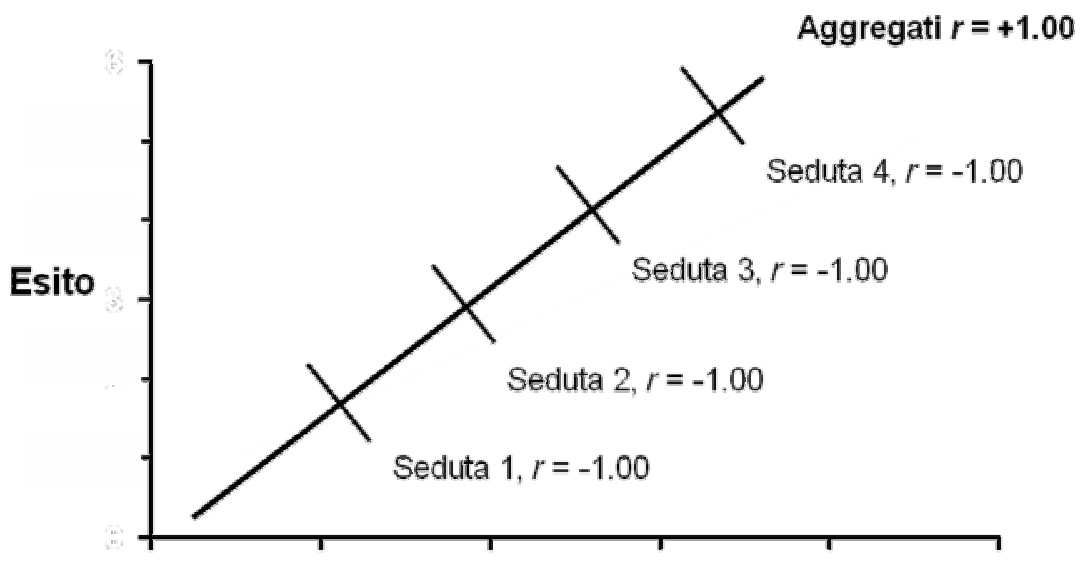

Interpretazioni del terapeuta

Figura 2. Esempio di come una correlazione che risulta positiva utilizzando dati aggregati tra le sedute possa nascondere una serie di correlazioni negative per ogni seduta (modificato da Shoham-Salomon, 1990).

2. Le analisi statistiche solitamente impiegate ci offrono solitamente un modello di cambiamento per il gruppo che non rispecchia il cambiamento dei singoli, anche nel caso di sofisticate procedure di analisi multivariata [che si svilupperanno a partire dai primi anni ' 80 ; si veda ad esempio Russell (1995)l. Questo è dovuto al fatto che la maggior parte delle analisi statistiche "tradizionali" - siano esse impiegate in disegni trasversali o longitudinali - considera la variabilità intra-individuale come errore, e dunque non permettono di generalizzare - come invece sempre si fa - dal gruppo studiato ai singoli individui che lo costituiscono (Molenaar, 2004; Molenaar \& Valsiner, 2008). Sarebbe dunque opportuno utilizzare procedure di analisi statistica che permettano innanzitutto di modellizzare il cambiamento a livello dei singoli individui e, solo successivamente, di produrre un modello generale al livello del gruppo. I cosidetti "modelli gerarchici di 
Ricerca in Psicoterapia / Research in Psychotherapy 2010; 1 (13): 61-91. http://www.researchinpsychotherapy.net analisi” rappresentano un'adeguata soluzione al riguardo (Gallop \& Tasca, 2009; Gelman \& Hill, 200\%; Tasca \& Gallop, 2009).

c) Si fa spesso uso di un arbitrario campionamento temporale - entro e tra le sedute - nella rilevazione delle variabili. Questo modo di procedere rispecchia l'assunto più o meno implicito che una o più variabili che misurano il processo terapentico abbiano lo stesso significato e la stessa rilevanza e importanza lungo il corso della terapia, per cui è legittimo studiare queste variabili solo in determinati occasioni. In realtà, in momenti diversi della terapia il medesimo processo può avere funzioni diverse, e differenti processi possono svolgere la medesima funzione. Ad esempio, eventuali silenzi del terapenta nelle fasi iniziali del trattamento possono avere la funzione di indurre il paziente ad aumentare la propria introspezione (ed essere quindi inoltre percepiti da quest'ultimo come controllanti), mentre in fasi successive della terapia, i medesimi silenzi possono consentire al paziente di elaborare materiale conflittuale precedentemente emerso, in un contesto di fiducia e mutuo rispetto (ed essere quindi percepiti come non controllanti).

Per uno studio adeguato del processo terapentico, le variabili di interesse devono essere misurate il più spesso possibile nel corso del trattamento. A tal proposito è opportuno osservare che, come già rilevato, dalla fine degli anni '70 emergerà una crescente tendenza da parte dei ricercatori a misurare e analizzare i dati il più frequentemente possibile.

d) Si fa spesso uso di unarbitraria suddivisione della terapia elo delle sedute. Quando si vuole studiare l'andamento delle variabili di interesse al passare del tempo, si è soliti suddividere arbitrariamente la terapia (quando le variabili vengono misurate dopo le sedute) o le sedute (quando le variabili vengono misurate all'interno dei trascritti delle sedute) in fasi, solitamente due o tre. Questo atteggiamento è problematico nella misura in cui la suddetta suddivisione è imposta dal ricercatore, e con molta probabilità non rappresenta un'adeguata finestra d'osservazione per esaminare la modificazione nel tempo delle variabili di processo studiate. Le fasi temporali in cui è possibile suddividere una seduta e/o un trattamento dovrebbero essere determinate attraverso una strategia di tipo bottom-up, in cui il ricercatore, dopo un'attenta esplorazione dei dati, si lasci "informare" da questi nello stabilire le fasi in cui è possibile suddividere una seduta e/o un trattamento (Gelo, Salvatore, \& Mergenthaler, in preparazione).

e) II processo terapentico è concepito come se fosse un fenomeno statico, cioè che non si modifica in funzione del passare del tempo. Questo assunto è evidente nell'utilizzo della "classica" statistica fisheriana per studiare il cambiamento di una o più variabili al passare del tempo (ad esempio, l'ANOVA e il $t$-test per misure ripetute così come l'analisi della regressione). ${ }^{25}$ Questo tipo di analisi non considera un fatto fondamentale, cioè che i valori delle variabili considerate si modificano al passare del tempo. Ciò è dovuto a due motivi. Primo, queste procedure statistiche si basano sull'assunto dell'indipendenza delle osservazioni, per cui i valori di una variabile rilevati al passare del tempo (ad esempio nel corso di una seduta e/o di un trattamento) sarebbero indipendenti gli uni dagli altri. Secondo, in questo tipo di procedure statistiche lo studio del cambiamento

\footnotetext{
${ }^{25}$ È opportuno qui ricordare che questo tipo di analisi viene utilizzato anche per lo studio del cambiamento nella ricerca di esito, per cui le osservazioni fatte qui sono valide anche per lo studio dell'esito terapeutico.
} 
Ricerca in Psicoterapia / Research in Psychotherapy 2010; 1 (13): 61-91. http://www.researchinpsychotherapy.net consiste essenzialmente nell'analisi di una serie successiva di differenze tra osservazioni temporali adiacenti (ad esempio, seduta $\rightarrow$ seduta $\rightarrow$ seduta3 $\rightarrow$...), per cui il cambiamento nel corso del tempo viene quantificato nei termini dell'entità dell'incremento dei valori di una variabile tra due misurazioni (Francis et al., 1991). In altri termini, il tempo è uno parametro d'osservazione, e la dipendenza delle variabili osservate da esso non può essere adeguatamente studiata.

Il processo terapeutico è per sua natura un fenomeno dinamico (cioè, tempo-dipendente), nella misura in cui si dispiega al passare del tempo. Non a caso la ricerca sul processo fa molto spesso uso di dati raccolti in diversi momenti che si succedono nel tempo $\left(t_{0} \rightarrow t_{1} \rightarrow t_{2} \rightarrow \ldots \rightarrow t_{n}\right)$. In questo tipo di dati, non è possibile considerare un'osservazione condotta nel momento $t_{n}$ come indipendente da unosservazione della stessa variabile in un momento $t_{n++}$, nella misura in cui, in questo caso, il valore di una variabile in $t_{\mathrm{n}}$ è necessariamente influenzata dal suo valore in $t_{n-l}$ e a sua volta influenza il suo valore in $t_{n+r}$. Di conseguenza, sarebbe opportuno utilizzare procedure di analisi longitudinale dei dati che tengano conto di questo fatto fondamentale. L'analisi delle curve di crescita o l'analisi delle serie temporali, ad esempio, rappresentano utili soluzioni a riguardo [Tschacher \& Ramseyer (2009); per una rassegna esaustiva si veda Singer \& Willet, (2003)].

f) Si predilige lo studio del rapporto tra variabili isolate (ad esempio, il rapporto tra la frequenza degli interventi supportivi del terapeuta e i livelli di insight del paziente). Molte delle analisi tradizionali (ad esempio indici di correlazione o di regressione) presuppongono l'adesione implicita ad un modello causale lineare del tipo: "se $x$, allora $y$. Nonostante esistano strategie di analisi (ad esempio, l'analisi della regressione multipla o la path analysis - che rappresenta una versione sofisticata della prima) che permettono di considerare rapporti più complessi tra diverse variabili, queste strategie presentano ancora il problema di essere applicate a dati aggregati tra i gruppi. Sarebbe invece più opportuno analizzare (e poi interpretare) sequenze e pattern temporali di variabili [per una discussione a riguardo si veda Gelo, Gennaro, \& Salvatore, (2008); Lauro-Grotto, Salvatore, Gennaro, \& Gelo (2009); Salvatore, Lauro-Grotto, Gennaro, \& Gelo (2009); Shoham-Salomon (1990)]. Per far ciò è necessario utilizzare appropriati disegni di tipo longitudinale o misto che permettano di studiare il rapporto multivariato tra diverse variabili in diversi momenti che si succedono nel tempo.

Come già affermato, il processo terapentico è infatti un fenomeno che si modifica varia nel tempo, e diversi processi assumono un significato diverso in diversi momenti della seduta e della terapia (contestualità). Di conseguenza, sono le diverse occorrenze di specifiche sequenze e pattern di variabili, e non le occorrenze di singole variabili, a poter indicare la significatività clinica di ciò che accade durante nel corso della terapia.

g) Si fa uso di un approccio esclusivamente quantitativo. Come nel caso della ricerca sull'esito, la ricerca sul processo viene condotta esclusivamente all'interno di un approccio esclusivamente quantitativo. Questo implica, come abbiamo visto nel corso del presente articolo, che 1) il processo terapentico venga misurato attraverso scale di misurazione che traducono le proprietà dei fenomeni osservati in quantità numeriche; 2) i dati vengano analizzati attraverso procedure statistiche; 3) la logica della ricerca aderisca a un modello ipotetico-deduttivo del fare scienza. Tuttavia, anche nella 
Ricerca in Psicoterapia / Research in Psychotherapy 2010; 1 (13): 61-91. http://www.researchinpsychotherapy.net ricerca sul processo, gli anni '90 saranno testimoni della crescente applicazione di metodologie e metodi di tipo qualitativo. Un approceio del genere è caratterizzato dal tentativo di sviluppare - e non di verificare modelli teorici attraverso unattenta e dettagliata analisi del materiale empirico considerato seguendo una logica essenzialmente abduttiva (Reichertz, 2009). I dati vengono solitamente raccolti attraverso interviste aperte o semistrutturate, offrendo il vantaggio di lasciar maggior "spazio" ai soggetti studiati (paziente e/o terapenta) di esprimere il proprio personale vissuto soggettivo relativamente a quanto avvenuto nel corso di una o più sedute. Il materiale testuale così raccolto viene poi analizzato attraverso specifiche analisi qualitative del testo, che permettono di identificare sia il contenuto sia la struttura di ciò che è stato espresso [per una rassegna si veda Frommer \& Rennie (2006) e, in questo numero, Blasi, (2010)].

\subsection{Rapporto tra processo ed esito}

a) Viene proposta una visione dicotomica dell'esito e del processo terapentico. Il primo viene infatti identificato con il cambiamento del paziente misurato quasi esclusivamente "al di fuori del trattamento" (prospettiva extra-therapy) in relazione a un qualche criterio clinicodiagnostico (si veda a tal proposito l'utilizzo di differenze pre-post-follow up per misurare l'entità dell'esito terapentico), mentre il processo terapeutico riguarda invece ciò che avviene "all'interno delle sedute" (prospettiva within-therapy).

In realtà, processo ed esito rappresentano due costrutti euristici che possono essere utili per individuare e descrivere alcune delle dimensioni essenziali della psicoterapia, ma che non devono per questo essere reificati. Inoltre, la pratica clinica ci insegna che, ciò che da una determinata prospettiva potrebbe essere considerato un aspetto del processo terapeutico, da un'altra prospettiva potrebbe rappresentare un aspetto dell'esito. Ciò è tanto più vero quanto più la scala d’osservazione si riduce lungo un continuum che va dal livello macro (la terapia e le fasi che la costituiscono) al livello micro (le sedute e i diversi momenti all'interno di esse), che porta quasi a far scomparire una netta distinzione tra ciò che è processo e ciò che è esito. Ad esempio, l’insight terapeutico osservato in specifici momenti di una o più sedute può essere considerato sia un aspetto del processo (ciò che succede nel corso della seduta) che un aspetto dell'esito (ciò che viene prodotto dagli avvenimenti che hanno luogo nel corso della seduta).

b) Lo studio dellesito e del processo sono indipendenti. Il fatto che l'esito e il processo terapeutico vengano considerati come due aspetti indipendenti della psicoterapia giustifica, di fatto, una rigida e netta separazione tra la ricerca sull'esito e la ricerca sul processo. È come se ciò che accade durante la terapia non avesse a che fare con l'esito osservato al termine di essa. Il tipo di ricerca empirica associata a questo modo di concepire l'esito e il processo terapeutico conduce così a dei risultati abbastanza sterili. La ricerca sull'esito, infatti, pur consentendoci di affermare che una terapia è efficace, nulla ci dice su meccanismi responsabili di tale efficacia. Al contrario, la ricerca sul processo ci permette di valutare cosa accade nel corso delle sedute, ma non è in grado di fornire informazioni relative alla significatività clinica di questi fenomeni.

Una scienza della psicoterapia che sia in grado di produrre conoscenza utile tanto per $\mathrm{i}$ ricercatori quanto per i clinici dovrebbe prefiggersi l'obiettivo di spiegare in che modo ciò che accade durante le sedute può o 
Ricerca in Psicoterapia / Research in Psychotherapy 2010; 1 (13): 61-91. http://www.researchinpsychotherapy.net meno produrre cambiamenti clinicamente significativi. Per far ciò, è necessario sviluppare strategie di ricerca che permettano di investigare in modo sistematico il rapporto tra processo ed esito terapeutico. In effetti, nel corso dei tre decenni considerati in questo articolo, si è iniziato ad osservare un crescente interesse per il rapporto tra il processo e l'esito terapentico, interesse che sarebbe poi esploso a partire dai primi anni '80. Ciò è avvenuto perché alcuni clinici e ricercatori - le cui figure tendevano col passare del tempo a coincidere sempre di più - hanno iniziato a interessarsi e a porsi domande sul "come e perché la terapia funziona". Ciò ha dato origine alla ricerca sul processo-esito, che rappresenta un primo tentativo di collegare il processo all'esito terapentico per cercare di valutare quali aspetti del processo giochino un ruolo nel determinare l'efficacia dei trattamenti [per una dettagliata rassegna e discussione a riguardo si veda Gelo, Manzo, Auletta \& Gennaro (submitted); Manzo, (2009)].

c) II cambiamento non ha a che fare con il processo terapentico. II "cambiamento" viene inteso esclusivamente nei termini di cambiamento clinico - e cioè nei termini della modificazione pre-post di specifiche variabili di esito. Al contrario, nonostante si faccia riferimento al processo terapeutico come a un qualcosa che si modifica nel corso delle sedute e/o del trattamento - e sono proprio queste modificazioni che vengono studiate dalla ricerca sul processo - tale modificazione viene raramente concettualizzata in stretto riferimento al cambiamento clinico. Il paradosso è che il processo terapeutico, che da una prospettiva clinica ha effettivamente a che fare col cambiamento, non viene - da una prospettiva teorico-metodologica e di analisi dei dati - considerato essere collegato ad esso.

\section{Conclusioni}

Lo studio empirico della psicoterapia si organizza, ai suoi inizi, attorno a due filoni principali: la ricerca sull'esito - tesa a dimostrare l'efficacia dei trattamenti psicoterapentici - e la ricerca sul processo - tesa a indagare cosa accade nel corso della psicoterapia. Il presente articolo si proponeva di passare in rassegna questi due indirizzi principali della ricerca sulla psicoterapia nel periodo che va dai primi anni '50 ai primi anni '80. La scelta di concentrarsi su questo specifico arco temporale è giustificata dal fatto che queste tre decadi rappresentano quello che può essere definito il paradigma classico della ricerca in psicoterapia, caratterizzato dalla necessità di dimostrare la possibilità di uno studio scientifico della psicoterapia attraverso l'adesione a un modello di scienza di stampo neopositivistico.

E stato possibile osservare come la ricerca sull'esito e sul processo terapentico siano caratterizzate da diversi approcei e disegni di ricerca, livelli e strumenti di osservazione, e analisi dei dati. La ricerca sull'esito, che intende valutare l'effettiva efficacia di uno o più trattamenti psicoterapentici nel produrre determinati cambiamenti, utilizza un approccio di ricerca sperimentale e/o quasi-sperimentale, che permette di valutare un eventuale rapporto di causa-effetto tra la somministrazione di uno o più trattamenti e la modificazione di determinate variabili di esito considerate essere indicatori attendibili del benessere clinico dei pazienti (come ad esempio la sintomatologia e/o la problematicità dei rapporti interpersonali). A tal fine, vengono utilizzati disegni di ricerca misti, che permettono di studiare gli effetti dell'intervento psicoterapentico su una o 
Ricerca in Psicoterapia / Research in Psychotherapy 2010; 1 (13): 61-91. http://www.researchinpsychotherapy.net più variabili di esito al passare del tempo in due o più gruppi. Queste variabili vengono misurate attraverso questionari standardizzati auto- e/o etero-somministrati all'inizio del trattamento (pre), al termine di esso (post) ed eventualmente in un periodo successivo al termine del trattamento (follow up). Infine, i dati vengono analizzati attraverso procedure di elaborazione statistica che consentono di verificare se esiste una differenza significativa tra $\mathrm{i}$ valori medi delle variabili di esito misurate rispettivamente nel corso del tempo e tra i gruppi. Il calcolo dell'effect size può inoltre essere utilizzato quando si vogliono "riassumere" quantitativamente i risultati di diversi studi di esito presenti in letteratura (meta-analisi).

A differenza della ricerca sull'esito, la ricerca sul processo intende invece studiare cosa accade nel corso di un trattamento psicoterapentico. A tal fine, fa uso di un approccio non sperimentale (detto anche passivo-osservazionale o correlazionale), che permette di esaminare il rapporto esistente tra diverse variabili ascrivibili al paziente, al terapenta e/o alla loro relazione. L'utilizzo di un approccio non sperimentale è dovuto all'estrema complessità del processo terapeutico, che non permette di soddisfare i criteri di controllo sperimentale se non al prezzo di uno "snaturamento" dell'oggetto di studio. Vengono impiegati principalmente disegni di ricerca trasversali - che permettono di studiare differenze tra gruppi (ad esempio tra i pazienti, tra i terapenti e/o tra pazienti e terapeuti) nelle variabili di processo esaminate e longitudinali - che permettono di studiare le variabili di interesse al passare del tempo in un solo gruppo (ad esempio, pazienti o terapeuti). In alcuni casi, tuttavia, si assiste all'impiego di disegni misti, in cui le variabili di interesse vengono esaminate in relazione a eventuali differenze tra $i$ gruppi e al passare del tempo. Le variabili considerate, che possono far riferimento a tutti quei fenomeni, avvenimenti e/o attivita ascrivibili al paziente, al terapenta e/o alla loro relazione nel corso di un trattamento (come ad esempio i livelli di empatia e/o di espressività emotiva, il comportamento verbale dei contraenti la relazione terapeutica, etc.), vengono generalmente misurate nel corso delle sedute - attraverso l'impiego di sistemi di codifica dei trascritti e/o dei video delle sedute - $o$ alla fine di esse - attraverso l'impiego di questionari solitamente auto-somministrati. Infine, i dati così ottenuti vengono analizzati attraverso procedure di analisi statistica che permettono di valutare il rapporto esistente tra una o più variabili misurate e la loro eventuale diversa distribuzione nel tempo.

Sono stati descritti e discussi una serie di assunti problematici che contraddistinguono questa prima fase storica della ricerca in psicoterapia, con particolare riferimento al modo di concettualizzare e analizzare l'esito e il processo terapentico. A livello generale, l'aspetto problematico principale consiste in una visione dicotomica dell'esito e del processo terapentico, secondo cui il primo coincide esclusivamente con gli effetti del trattamento misurati al termine di esso, mentre il secondo riguarda esclusivamente ciò che accade nel corso della psicoterapia. In realtà, questi due costrutti non sono ontologicamente differenti, ma "semplicemente" rappresentano delle euristiche che possono essere utili per individuare e descrivere due delle dimensioni essenziali della psicoterapia. Nella pratica clinica, queste due dimensioni sono strettamente interconnesse, per cui ciò che rappresenta il processo e ciò che rappresenta l'esito dipendono dalla prospettiva assunta dall'osservatore. Si è visto ad esempio infatti come, passando da una prospettiva di osservazione macro-analitica a una micro-analitica, le differenze tra processo ed esito tendono a diventare sempre più sfumate, per cui ciò che può essere considerato processo da una prospettiva, può legittimamente essere considerato esito da un'altra (si pensi ad esempio al 
Ricerca in Psicoterapia / Research in Psychotherapy 2010; 1 (13): 61-91. http://www.researchinpsychotherapy.net concetto di insight visto come processo essenziale per raggiungere e produrre il cambiamento oppure come esito di un cambiamento avvenuto a qualche altro livello).

Tale assunto sulla natura del processo e dell'esito è ciò che, fondamentalmente, ha condotto - in queste prime fasi storiche della ricerca in psicoterapia - a una rigida distinzione tra la ricerca sul processo e la ricerca sull'esito, limitando il tipo di risposte che era possibile ottenere relativamente alla pratica psicoterapeutica. La ricerca sull'esito, infatti, pur consentendoci di affermare che una terapia è efficace, non è in grado di fornire informazioni sui meccanismi sottostanti questa eventuale efficacia; la ricerca sul processo, al contrario, permette di valutare cosa accade nel corso delle sedute, ma non è in grado di fornire informazioni relative all'efficacia clinica di questi fenomeni.

A livello più specifico, un assunto problematico consiste nel fatto che il cambiamento - riguardi esso variabili di processo e/o di esito - viene considerato un fenomeno statico, omogeneo e uniforme tra ed entro i soggetti e nel corso delle sedute [si veda a tal proposito il mito dell'uniformità descritto da Kiesler (1966)]. Tale assunto, chiaramente contraddetto dalla pratica clinica, si manifesta nella scelta "economica" di misurare le variabili di esito e/o di processo in un numero limitato di occasioni al passare del tempo, ed è problematico perché non permette di osservare con precisione quanto accade tra i diversi momenti temporali. Inoltre, tale assunto si riflette nell'utilizzo di procedure di analisi longitudinali dei dati che non permettono di studiare l'effettiva natura dinamica del processo e dell'esito terapeutico. In questo tipo di analisi, infatti, il tempo è considerato semplicemente un parametro di osservazione, e non una variabile presa in considerazione all'interno delle analisi stesse.

Un altro assunto problematico riguarda la convinzione che il cambiamento (sia esso relativo a variabili di processo e/o di esito) possa essere adeguatamente studiato prendendo in considerazione prestazioni di "gruppo". Tale assunto, che è alla base di tutta la ricerca psicosociale tradizionale, si riflette nell'utilizzo di procedure statistiche che: 1) analizzano il cambiamento nei valori di una o più variabili in riferimento alla variabilità inter-individuale; 2) utilizzano dati aggregati tra o entro i soggetti; 3) in cui la variabilità intra-individuale viene considerata errore. Come conseguenza di ciò, i risultati ottenuti in questo modo ci offrono un modello di cambiamento per il gruppo che non rispecchia necessariamente il cambiamento a livello dei singoli. In altri termini, le differenze individuali vengono "appiattite", rendendo di fatto impossibile generalizzare i risultati ottenuti sul gruppo ai singoli individui di cui il gruppo è costituito (Molenaar, 2004).

Infine, un ultimo aspetto problematico riguarda l'assunto secondo il quale il paradigma quantitativo rappresenti l'unico adeguato approccio per lo studio della psicoterapia. Ciò è dovuto alla necessità, da parte della nascente ricerca in psicoterapia, di avvalersi delle metodologie d'indagine tradizionalmente accettate come valide nell'ambito delle scienze esatte, con il fine di fornire legittimazione scientifica e sociale allo studio empirico della psicoterapia. L'utilizzo di un approccio esclusivamente quantitativo si rivela problematico nella misura in cui esso, riflettendo un'epistemologia oggettivista, realista e meccanicistica, si traduce in metodologie di indagine riduzionistiche che, orientate da una logica della giustificazione, hanno come obiettivo quello di spiegare (Erklären) i fenomeni studiati, trascurando la dimensione della comprensione (Verstehen).

I problemi di natura concettuale e metodologica descritti sopra - che rappresentano quello che è stato definito il paradigma classico della ricerca 
Ricerca in Psicoterapia / Research in Psychotherapy 2010; 1 (13): 61-91. http://www.researchinpsychotherapy.net

in psicoterapia - verranno tuttavia recepiti dalla comunità scientifica che, a partire dai primi anni ' 80 , si adopererà per cercare, almeno in parte, di farvi fronte. Emergerà innanzitutto la consapevolezza che, per comprendere effettivamente la psicoterapia, è necessario sviluppare strategie di ricerca che permettano di indagare in modo sistematico il rapporto tra processo ed esito. Ciò si rifletterà nel consolidarsi della ricerca di processo-esito (Gelo et al., submitted, Greenberg, 1991) e nella nascita della ricerca sul processo di cambiamento (Orlinsky et al., 2004; Gelo, Manzo, Auletta, \& Gennaro, in preparazione). Da un punto di vista metodologico, si assisterà: 1) a una sofisticazione dei disegni e delle procedure di analisi statistica - soprattutto nella direzione di garantire un più adeguato studio dei dati longitudinali che non considerano la variabilità intra-individuale come errore [per una sintetica trattazione si veda Gallop \& Tasca (2009); Tasca \& Gallop (2009)]; 2) a un utilizzo sempre crescente degli approcei qualitativi che, fondandosi su un'epistemologia di stampo fenomenologico, ermenentico e sociocostruttivista, permetteranno di studiare sempre più la psicoterapia secondo una logica della scoperta, dando rilevanza alla comprensione dei vissuti e delle esperienze dei contraenti la relazione terapentica.

Per concludere l'incontro psicoterapentico tra paziente e terapeuta è un fenomeno estremamente articolato, multiforme e complesso, e lo studio empirico della psicoterapia rappresentare senza ombra di dubbio un compito arduo e difficile. Con il presente articolo speriamo di essere riusciti a illustrare sia le caratteristiche che $\mathrm{i}$ problemi principali che hanno caratterizzato la ricerca in psicoterapia nelle sue fasi iniziali.

\section{Bibliografia}

Barkham, M., Stiles, W.B., \& Shapiro, D.A. (1993). The shape of change in psychotherapy: Longitudinal assessment of personal problems. Journal of Consulting and Clinical Psychology, 61, 4, 667-67\%.

Barlow, D.H., \& Hersen, M. (1984). Single Case Experimental Designs: Strategies for Studying Behavior Change (2th ed.). New York: Allyn \& Bacon.

Barrett-Lennard, G.T. (1962). Dimensions of therapist response as causal factors in therapeutic change. Psychological Monographs: General and Applied, 86, 1-36.

Beck, A.T., Ward, C.H., Mendelson, M., Mock, J., \& Erbaugh, J. (1961). An inventory for measuring depression. Archives of General Psychiatry, 4, $561-71$.

Beck, A.T., Steer, R.A., \& Brown, G.K. (1996). Manual for the Beck Depression Inventory-II. San Antonio, TX: Psychological Corporation.

Benjamin, L.S. (1974). Structural analysis of social behavior. Psychological Review, 81, 392-425.

Bergin, A.E., \& Garfield, S.L. (Eds.). (1986). Handbook of psychotherapy and behavior change (3th ed.). New York: Wiley.

Bergin, A.E., \& Garfield, S.L. (Eds.). (1994). Handbook of Psychotherapy and Behavior Change (4th ed.) New York: Wiley.

Bernard, H.R. (2009). Analyzing Qualitative Data: Systematic Approaches. Sage Publications.

Blasi, S. (2010). La ricerca qualitativa in psicoterapia. Controversie, applicazioni e criteri di qualità. Ricerca in Psicoterapia, Research in psychotherapy: psychopathology, Process and outcome, 1, 23-60. 
Ricerca in Psicoterapia / Research in Psychotherapy 2010; 1 (13): 61-91.

http://www.researchinpsychotherapy.net

Bond, M. (1986). Defense Style Questionnaire. In G.E. Vaillant (Eds.), Empirical Studies of Ego Mechanisms of Defense (pp. 146-152). Washington, DC: American Psychiatric Press.

Borkovec, T.D., \& Sibrava., N.J. (2005). Problems with the use of placebo conditions in psychotherapy research, suggested alternatives, and some strategies for the pursuit of the placebo phenomenon: The placebo concept in psychotherapy. Journal of clinical psychology, 61(7), 805-818.

Collins, L.M., \& Sayer, A.G. (Eds.). (2001). New Methods in the Analysis of Change Washington, DC: APA Publications.

Corbetta, P. (2003). La Ricerca sociale: Metodologia e tecniche. I. I paradigmi di riferimento. Bologna: Il Mulino.

Crits-Christoph, P. (1997). Limitations of the Dodo Bird Verdict and the role of clinical trials in psychotherapy research: Comment on Wampold et al. (1997). Psychological Bulletin, 122(3), 216-220.

Dazzi, N. (2006). Il dibattito contemporaneo sulla ricerca in psicoterapia. In N. Dazzi, V. Lingiardi \& A. Colli (Eds.), La ricerca in psicoterapia. Modelli e strumenti (pp. 3-30). Milano: Cortina.

Del Rio, M.T., \& Molina, M.E. (2008). Nomothetic and idiographic approaches: Constructing a bridge. In S. Salvatore, J. Valsiner, S. StroutYagodzynski \& J. Clegg (Eds.), Yearbook of Idiographic Science, Volume 1 (pp. 75-80). Rome: Firera \& Liuzzo Group.

Derogatis, L.R. (1978). SCL_90-R: Administration, Scoring and Procedures Manual-I for the R(evised) Version. Baltimore: The Johns Hopkins University Press.

Derogatis, L.R. (1992). SCL_90-R: Administration, Scoring and Procedures Manual-II. Baltimore, MD: Clinical Psychometric Research.

Dittes, J.E. (1959). Previous studies bearing on content analysis of psychotherapy. In J. Dollard \& F. Auld (Eds.), Scoring Human Motives: A Manual (pp. 325-351). New Haven, CT: Yale University Press.

Elliott, R. (2002). Hermeneutic single case efficacy design. Psychotherapy Research, 12(1), 1-21.

Elliott, R., \& Anderson, C. (1994). Simplicity and complexity in psychotherapy research. In R.L. Russell (Ed.), Reassessing Psychotherapy Research (pp. 65-113). New York: Guilford Press.

Evans, C., Mellor-Clark, J., Margison, F., Barkham, M., McGrath, G., Connell, J., \& Audin, K. (2000). Clinical Outcomes in Routine Evaluation: The CORE-OM. Journal of Mental Health, 9, 247-255.

Eysenck, H.J. (1952). The effects of psychotherapy: An evaluation. Jonrnal of Consulting Psychology, 16, 319-324.

Fielder, F.E. (1951). A method of objective quantification of certain counter-transference attitudes. Journal of Clinical Psychology, \%, 101$10 \%$.

Francis, D.J., Fletcher, J.M., Stuebing, K.K., Davidson, K.C., \& Thompson, N.M. (1991). Analysis of change: Modeling individual growth. Journal of Consulting e Clinical Psychology, 59, 27-37.

Freni, S., \& Papini, S.P. (2006). Una rassegna critica degli strumenti utili per la ricerca in psicoterapia. In N. Dazzi, V. Lingiardi \& A. Colli (Eds.), La ricerca in psicoterapia. Modelli e strumenti (pp. 91-122). Milano: Cortina.

Freud, S. (1892-95). Studi sullisteria. OSF, vol. I.

\footnotetext{
${ }^{*}$ Salvo diversa indicazione, per la traduzione italiana degli scritti di Sigmund Freud si fa riferimento alle "Opere", edite da Boringhieri, Torino 1967-1980, in 12 volumi, che citiamo con OSF e numero del volume.
} 
Ricerca in Psicoterapia / Research in Psychotherapy 2010; 1 (13): 61-91. http://www.researchinpsychotherapy.net

Freud, S. (1909). Osservazioni su un caso di nevrosi ossessiva (Caso clinico dell'nomo dei topi). OSF, vol. VI.*

Friedlander, M.L. (1982) Counseling discourse as a speech event: Revision and extension of the Hill Counselor Verbal Response Category System. Journal of Counseling Psychology, 29, 425-429.

Frommer, J., \& Rennie, D.L. (2006). Qualitative Psychotherapy Research Methods and Methodology. Lengerich: Pabst.

Gallop, R., \& Tasca, G.A. (2009). Multilevel modeling of longitudinal data for psychotherapy researchers: II. The complexities. Psychotherapy Research, 19(4-5), 438-45\%.

Gelman, A., \& Hill, J. (200\%). Data Analysis Using Regression and Multilevel/Hierarchical Models. Cambridge: Cambridge University Press.

Gelo O., Braakmann, D., \& Benetka, G. (2008). Quantitative and Qualitative Research: Beyond the Debate. Integirative Psychological and Behavioural Science, 42(3), 266-90.

Gelo, O., Gennaro, A., \& Salvatore, S. (2007). Per un approccio dinamico allo studio del processo terapentico. Psicoterapia, 34-35, 159-169.

Gelo, O., Manzo, S., Auletta, A., \& Gennaro, A. (submitted). Aspetti teoricometodologici e analisi dei dati nella ricerca in psicoterapia. Parte II: La ricerca sul processo-esito - dagli anni '50 ad oggi.

Gelo, O., Manzo, S., Auletta, A., \& Gennaro, A. (2010). Aspetti teoricometodologici e analisi dei dati nella ricerca in psicoterapia PARTE III: La ricerca sul processo di cambiamento - dagli anni '80 ad oggi. Ricerca in Psicoterapia, Research in psychotherapy: psychopathology, Process and outcome, 1, 61-9\%.

Gelo, O., Salvatore, S., \& Mergenthaler, E. (in preparazione). A paradigm for single-case quantitative research: The Therapentic Cycle Model in a metacognitive-interpersonal psychotherapy.

Greenberg, L.S. (1986). Change process research. Journal of Consulting and Clinical Psychology, 54(1), 4-9.

Greenberg, L.S. (1991). Research on the process of change. Psychotherapy Research, I(1), 3-16.

Greenberg, L.S. (2007). A guide to conducting a task analysis of psychotherapeutic change. Psychotherapy Research, 1\%, 15-30.

Greenberg, L.S., \& Newman, F.L. (1996). An approach to psychotherapy change process research: Introduction to the special section. Journal of Consulting and Clinical Psychology, 64(3), 435-438.

Greenberg, L.S., \& Pinsof, W.M. (Eds.). (1986). The Psychotherapentic Process: A Research Handbook. New York: Guilford Press.

Guerney, B.G. (1978). Relationship Enhancement: Skill-Training Programs for Therapy, Üproblem Prevention, and Enrichment. San Francisco: Jossey-Bass.

Hartley, D.E., \& Strupp, H.H. (1983). The therapentic alliance: its relationship tout come in brief psychotherapy. In J. Masling (Ed.), Empirical Studies of Psychoanalytical Theories (pp. 1-38). Hillsdale, NJ: Analytical Press.

Harway, N.I., Dittman, A.T., Raush, H.L., Bordin, E.S., \& Rigler, D. (1955). The measurement of depth of interpretation. Journal of Consulting Psychology, 19, 247-253.

Hedlund, J.L., \& Viewig, B.W. (1979). The Hamilton Rating Scale for Depression: A comprehensive Review. Journal of Operational Psychiatry, 10, 149-165. 
Ricerca in Psicoterapia / Research in Psychotherapy 2010; 1 (13): 61-91.

http://www.researchinpsychotherapy.net

Hill, C.E. (1978) Development of a Counselor Verbal Response Category System. Journal of Counseling Psychology, 25, 461-468.

Hill, C.E., \& Lambert, M.J. (2004). Methodological issues in studying psychotherapeutic processes and outcome. In M.J. Lambert (Ed.), Bergin and Garfield's Handbook of Psychotherapy and Behaviour Change (5th ed.) (pp. 55-71). New York: Wiley.

Hill, C.E., Thames, T.B., \& Rardin, D.K. (1979). Comparison of Rogers, Perls and Ellis on the Hill Counselor Verbal Response Category System. Journal of Counselling Psychology, 26(3), 198-203.

Hill, C.E., Thompson, B.J., Cogar, M.C., \& Denman, D.W. (1993). Beneath the surface of long-term therapy: therapist and client report of their own and each other's covert processes. Journal of Counselling Psychology, $40(3), 278-28 \%$.

Hilliard, R.B. (1993). Single-case methodology in psychotherapy process and outcome research. Journal of Consulting and Clinical Psychology, 61(3), 373-380.

Horowitz, L.M., Rosenberg, S.E., Baer, B.A., Ureño, G., \& Villaseñor, V.S. (1988). Inventory of interpersonal problems: psychometric properties and clinical applications. Journal of Consulting and Clinical Psychology, 56, 885-892.

Huddleson, J.H. (1927). Psychotherapy in two hundred cases of psychoneurosis. The Military Surgeon, 60, 161-170.

Jones, M.C. (1924a). A laboratory study of fear: The case of Peter. Pedagogical Seminary, 31, 308-315.

Jones, M.C. (1924b). The elimination of children's fears. Journal of Experimental Psychology, \%, 382-390.

Kazdin, A.E. (1994). Methodology, design, and evaluation in psychotherapy research. In A.E. Bergin, \& S.L. Garfield (Eds.), Handbook of Psychotherapy and Behavior Change (4th ed.) (pp. 19-61). New York: Wiley.

Kiesler, D.J. (1966). Some myths of psychotherapy research and the search for a paradigm. Psychological Bulletin, 65, 110-136.

Kiesler, D.J. (1973). The Process of Psychotherapy: Empirical Foundations and Systems of Analysis. Chicago: Aldine.

Kiesler, D.J. (1983). The Paradigm Shift in Psychotherapy Process Research. Unpublished manuscript.

Klein, M.J., \& Elliott, R. (2006). Client accounts of personal change in process-experiential psychotherapy: A methodologically pluralistic approach. Psychotherapy research, 16, 91-105.

Klein, M.H., Mathieu, P.L., Gendlin, E.T., \& Kiesler, D.J. (1969). The Experiencing Scale: A Research and Training Manual. Madison, Wisconsin: Wisconsin Psychiatric Institute.

Knight, R.P. (1941). Evaluation of the results of psychoanalytic therapy. The American Journal of Psychiatry, 98, 434-446.

Kolden, G.G. (1991). The generic model of psychotherapy: An empirical investigation of patterns of process and outcome relationships. Psychotherapy Research, 1(1), 62-73.

Kuhn, T.S. (1962). The structure of Scientific Revolution. Chicago: University of Chicago Press. Tr. it. La struttura delle rivoluzioni scientifiche. Torino: Einandi, 1969.

Kuhnlein I. (1999). Psychotherapy as a process of transformation: The analysis of post therapeutic autobiographical narrations. Psychotherapy research,.$(3)$, $2 \% 4-288$.

Lambert, M.J. (Ed.). (2004). Bergin and Garfield's Handbook of Psychotherapy and Behavior Change (5th ed.). New York: Wiley. 
Ricerca in Psicoterapia / Research in Psychotherapy 2010; 1 (13): 61-91.

http://www.researchinpsychotherapy.net

Lambert, M.J., \& Archer, A (2007). I risultati della ricerca sugli effetti della psicoterapia e le loro conseguenze per la pratica clinica. Dove la pratica e la ricerca si incontrano. In C.D. Goodhearth \& A.E. Kazdin (Eds.), Psicoterapia a prova di evidenza (pp. 113-134).

Lambert, M.J., \& Bergin, A.E. (1994). The effectiveness in psychotherapy. In A.E. Bergin e S.L. Garfield (Eds.), Handbook of Psychotherapy and Behavior Change (4th ed.) (pp. 143-189). New York: Wiley.

Lambert, M.J. \& Ogles, B.M. (2004). The efficacy and effectiveness of psychotherapy. In M.J. Lambert (Ed.), Handbook of Psychotherapy and Behavior Change (5th ed.) (pp. 139-193). New York: Wiley.

Lambert, M.J., Morton, J., Hatfield, D., Harmon, C., Hamilton, S., Reid, R., Shimokawa, K., Christopherson, C.D., \& Burlingame, G. (2004). Administration and Scoring Manual for the Outcome Questionnaire $(O Q$-45.2) (3th ed.). Wilmington, DE: American Professional Credentialling Services LLC.

Lauro-Grotto, R., Salvatore, S., Gennaro, A., \& Gelo, O. (2009). The unbearable dynamicity of psychological processes: highlights from psychodynamic theories. In J. Valsiner, P.C. Molenaar, M. Lyra, \& N. Chaudhary (Eds.), Dynamic Process Methodologies in the Social and Developmental Sciences (pp. 1-30). New York: Springer.

Leitenberg, H., Wincze, J.P., Butz, R.A., \& Callahan, E.J. (1970).Comparison of the effects of instructions and reinforcement in the treatment of a neurotic avoidance response: A single case experiment. Journal of Behavior Therapy and Experimental Psychiatry, 1(1), 53-58.

Lepper, G., \& Riding, N. (2006). Researching the Psychotherapy Process: A Practical Guide to Transcript-Based Methods. Basingstoke, Hampshire: Palgrave Macmillan.

Lingiardi, V. (2006). La ricerca single-case. In N. Dazzi, V. Lingiardi \& A. Colli (Eds.), La ricerca in psicoterapia. Modelli e strumenti (pp. 111131). Milano: Cortina.

Loose, J. (2009). Filosofia della scienza. Unintroduzione. Tr. it. Il Saggiatore, Milano 2009.

Luborsky, L. (1976). Helping alliance in psychotherapy. In J.L. Cleghorn (Ed.), Succesful Psychotherapy (pp. 92-116). New York: Brunnel-Mazel.

Luborsky, L., Crits-Christoph, P., Alexander, L., Margolis, M., \& Cohen, M. (1983). Two helping alliance methods for predicting outcomes of psychotherapy: A counting signs versus a global rating method. Journal of Nervous and Mental Disease, 181, 480-492.

Luborsky, L., Singer, B., \& Luborsky, L. (1975). Comparative studies of psychotherapy: Is it true that "everyone has won and all must have prizes?". Archives of General Psychiatry, 32, 995-1008.

Manzo, S. (2010). La ricerca di processo nel decennio 1998-200\%: Contributo ad una review critica. Ricerca in Psicoterapia, Research in psychotherapy: psychopathology, Process and outcome, 1, 98-12\%.

Marsden, G. (1971) Content analysis studies in psychotherapy: 1954 through 1968. In A.E. Bergin \& S.L. Garfield (Eds.), Handbook of Psychotherapy and Behavior Change (1th ed.) (pp. 345-40\%). London: Wiley.

Meehl, P.E. (1978). Theoretical risks and tabular asterisks: Sir Karl, Sir Ronald, and the slow progress of soft psychology. Journal of Consulting and Clinical Psychology, 46(4), 806-834.

Meltzer, H. (1930). Present status of experimental studies on the relationship of feeling to memory. Psychological Review, 3\%, 124-139. 
Ricerca in Psicoterapia / Research in Psychotherapy 2010; 1 (13): 61-91.

http://www.researchinpsychotherapy.net

Mergenthaler, E. (1996). Emotion-abstraction patterns in verbatim protocols: A new way of describing psychotherapeutic processes. Journal of Consulting and Clinical Psychology, 64(6), 1306-1315.

Mergenthaler, E., \& Gelo, O. (2007). Unanalisi qualitativa del disturbo di personalità narcisista attraverso il Modello del Ciclo Terapentico: Uno studio single-case (Caso K.). In G. Nicolò \& S. Salvatore (Eds.), La ricerca sui risultati e sul processo in psicoterapia (pp. 313-327). Roma: Edizioni Carlo Amore.

Migone, P. (2006). Breve storia della ricerca in psicoterapia. In N. Dazzi, V. Lingiardi \& A. Colli (Eds.). La ricerca in psicoterapia. Modelli e strumenti (pp. 5-46). Milano: Cortina.

Milbrath, C., Bond, M., Cooper, S., Znoj, H., Horowitz, M.J., \& Perry, J.C. (1998). Sequential consequences of therapist interventions. Journal of Psychotherapy Practice and Research, 40(8), 40-54.

Molenaar, P. (2004). A manifesto on Psychology as idiographic science: Bringing the person back into scientific psychology, this time forever. Measurement, $2(4), 201-218$.

Molenaar, P., \& Valsiner, J. (2008). How generalization works through single case: A simple idiographic process analysis of an individual psychotherapy. In S. Salvatore, J. Valsiner, S. Strout-Yagodzynski \& J. Clegg (Eds.), YIS: Yearbook of Idiographic Science (Vol. 1) (pp. 23-38). Rome: Firera \& Liuzzo Group.

Morgan, G.A. (2006). Understanding and Evaluating Research in Applied Clinical Settings. Mahwah: Lawrence Erlbaum.

Norcross, J.C. (Eds.). (2002). Psychotherapy Relationships that Work. New York: Oxford University Press.

Noreross, J.C., \& Goldfried, M.R. (Eds.). (2005). Handbook of Psychotherapy Integration (2th ed.). New York: Oxford University Press.

Orlinsky, D.E., \& Howard, K.I. (1978). The relation of process to outcome in psychotherapy. In S. Garfield \& A. Bergin (Eds.), Handbook of Psychotherapy and Behavior Change, (2th ed.) (pp. 23-62). New York: Wiley.

Orlinsky, D.E., \& Howard, K.I. (1986). The psychological interior of psychotherapy: explorations with the therapy session reports. In L.S. Greenberg \& W.M. Pinsof (Eds.), The Psychotherapentic Process: A Research Handbook (pp. 325-366). New York: Guilford Press.

Orlinsky, D.E., \& Howard, K.I., (1987) A generic model of psychotherapy. Journal of Integrative and Eclectic Psychotherapy, 6, 6-2\%.

Orlinsky, D.E., \& Russell, R.L. (1994). Tradition and change in psychotherapy research: Notes in the fourth generation. In R.L. Russell (Ed.), Reassessing Psychotherapy Research (pp. 185-214). New York: Guilford Press.

Orlinsky, D.E., Grawe, K., \& Parks, B.K. (1994). Process and outcome in psychotherapy - noch Einmal. In A.E. Bergin \& S.L. Garfield (Eds.), Handbook of Psychotherapy and Behavior Change (4th ed.) (pp. 270376). New York: Wiley.

Orlinsky, D.E., Rønnestad, M.H., \& Willutzki, U. (2004). Fifty years of psychotherapy process-outcome research: Continuity and change. In Lambert, M. (Ed.), Bergin and Garfield's Handbook of Psychotherapy and Behavior Change (5th ed.) (pp. 307-392). New York: Wiley.

Reichertz, J. (2009). Abduction: The Logic of Discovery of Grounded Theory. Forum Qualitative Sozialforschung/Forum: Qualitative Social Research, 11, 1, art. 13, http://nbnresolving.de/urn:nbn:de:0114 fqs1001135. 
Ricerca in Psicoterapia / Research in Psychotherapy 2010; 1 (13): 61-91.

http://www.researchinpsychotherapy.net

Rice, L., \& Greenberg, L. (1984). Patterns of Change. New York: Guilford Press.

Riley, R.D., Lambert, P.C., Staesserr, J.A., Wang, J., Gueyffler, F., Thijs, L., \& Boutitie, F. (2008). Meta-analysis of continuous outcomes combining individual patient data and aggregate data. Statistics Medicine, 2\%, 1870-1893.

Rogers, C.R. (1942). Counseling and Psychotherapy. Boston: Houghton Mifflin.

Rogers, C.R. (1951). Client-centered Therapy: Its Current Practice, Implications, and Theory. Boston: Houghton Mifflin Company.

Rogers, C.R. (1975). Empathic: An unappreciated way of being. Counseling Psychologist, 5, 2-10.

Rogers, C.R. (195\%). The necessary and sufficient conditions of therapentic personality change. Journal of Consulting Psychology, 21, 95-103.

Rosenzweig, S. (1954). A transvaluation of psychotherapy: A reply to Hans Eysenck. The Journal of Abnormal and Social Psychology, 49(2), 298304.

Russell, R.L. (1995). Introduction to the special section on multivariate psychotherapy process research: Structure and change in the talking cure. Journal of Consulting and Clinical Psychology, 63(1), 3-5.

Russell, R.L., \& Trull, T.J. (1986). Sequential analysis of language variables in psychotherapy. Journal of Consulting and Clinical Psychology, 54, 1621.

Safran, J.D., Greenberg, L.S., \& Rice, L.N. (1992). Integrating psychotherapy research and practice: Modeling the change process. Psychotherapy, 25, 1-1\%.

Salvatore, S., \& Valsiner, J. (2008). Idiographic science on its way: Towards making sense of psychology. In S. Salvatore, J. Valsiner, S. StroutYagodzynski \& J. Clegg (Eds.), YIS: Yearbook of Idiographic Science (Vol. 1) (pp. 10-19). Rome: Firera \& Liuzzo Group.

Salvatore, S., Gelo, O., Gennaro, A., Manzo, S., \& Radaideh, A (in stampa). Looking at the psychotherapy process as an intersubjective dynamic of meaning- making. A case study with the Discourse Flow Analysis. Journal of Constructivist Psychology.

Salvatore, S., Lauro-Grotto, R., Gennaro, A., \& Gelo, O. (2009). Attempts to grasp the dynamicity of intersubjectivity. In J. Valsiner, P.C. Molenaar, M. Lyra \& N. Chaudhary (Eds.) Dynamic Process Methodologies in the Social and Developmental Sciences (pp. 171-190). New York: Springer.

Semerari A., Carcione A., Dimaggio G., Falcone M., Nicolò G., Procacci M. \& Alleva G. (2003). How to evaluate metacognitive functioning in psychotherapy? The Metacognition Assessment Scale and its applications. Clinical Psychology and Psychotherapy, 10, 238-261.

Shapiro, M.B., (1961). A method of measuring changes specific to the individual psychiatric patient. British Journal of Medical Psychology, 34, 151-155.

Shoham-Salomon, V. (1990). Interrelating research processes of process research. Journal of Consulting and Clinical Psychology, 58, 295-303

Singer, J.D., \& Willett, J.B. (2003). Applied Longitudinal Data Analysis: Modeling Change and Event Occurrence. New York: Oxford University Press.

Sloane, R.B., Staples, F.R., Cristol, A.H., Yorkston, N.J., \& Whipple, K. (1975). Psychotherapy versus Behaviour Therapy. Cambridge, MA: Harvard University Press.

Smith, M.L., \& Glass, G.V. (1977). Meta-analysis of psychotherapy outcome studies. American Psychologist, 32, 752-760. 
Ricerca in Psicoterapia / Research in Psychotherapy 2010; 1 (13): 61-91. http://www.researchinpsychotherapy.net

Smith, M.L., Glass G.V., \& Miller, T.I. (1980). The Benefits of Psychotherapy. Baltimore: The Johns Hopkins University Press.

Stiles, W.B. (1978) Verbal response modes and dimensions of interpersonal roles: A method of discourse analysis. Journal of Personality and Social Psychology, 36, 693-703.

Stiles, W.B. (1979) Verbal response modes and psychotherapeutic technique. Psychiatry, 42, 49-62.

Stiles, W.B., \& Shapiro, D.A. (1994). Disabuse of the drug metaphor: Psychotherapy process-outcome correlations. Journal of consulting and clinical psychology, 62, 942-948.

Stiles, W.B., Honos-Webb, L., \& Knobloch, M.M. (1999). Treatment process research methods. In P.C. Kendall, J.N. Butcher \& G.N. Holmbeck (Eds.), Handbook of Research Methods in Clinical Psychology (pp. 364402). New York: Wiley.

Stiles, W.B., Shapiro, D.A., \& Elliott, R. (1986). Are all psychotherapies equivalent? American Psychologist, 41, 165-180.

Tasca, G.A., \& Gallop, R. (2009). Multilevel modeling of longitudinal data for psychotherapy researchers: I. The basics. Psychotherapy Research, $19(4-5), 429-43 \%$.

Task Force on Promotion and Dissemination of Psychological Procedures (1995). Training in and dissemination of empirically validated psychological treatments: Reports and recommendations. The Clinical Psychologist, 48, 3-23.

Truax, C.B., \& Carkhuff, R.R. (196\%). Toward effective counseling and psychotherapy: Training and practice. Chicago: Aldine.

Tschacher, W., \& Ramseyer, F. (2009). Modeling psychotherapy process by time-series panel analysis (TSPA). Psychotherapy Research, 1X(4), 469481.

Tschacher, W., Baur, N., \& Grawe, K. (2000). Temporal interaction of process variables in psychotherapy. Psychotherapy Research, 10(3), 296309.

Valsiner, J., Molenaar, Peter, C.M., Lyra, M.C.D.P., \& Chandary, N. (Eds.) (2009). Dynamic Process Methodology in the Social and Developmental sciences. New York: Springer.

Voelkle, M.C. (200\%). Latent growth curve modeling as an integrative approach to the analysis of change. Psychology Science, 4X(4), 375-414.

Wampold, B.E. (2001). The Great Psychotherapy Debate: Models, Methods, and Findings. Mahwah, NJ: Lawrence Erlbaum.

Wampold, B.E., Mondin, G.W., Moody, M., Stich, E., Benson, K., \& HyunNie, A. (1997). A meta-analysis of outcome studies comparing bona fide psychotherapies: Empirically, "all must have prizes". Psychological Bulletin, 122, 203-215.

Wilson, G.T., Fairburn, C.C., Agras, W.S., Walsh, B.T., \& Kraemer, H. (2002). Cognitive-Behavioral Therapy for Bulimia Nervosa: Time course and mechanisms of change. Journal of Consulting and Clinical Psychology, 20, 26\%-274.

Wolpe, J. (1958). Psychotherapy by Reciprocal Inhibition. Stanford: Stanford University Press.

Zennaro, A., Cristofanelli, S., \& Vottero Ris, F. (2006). Indicazioni per una buona metodologia della ricerca. In N. Dazzi, V. Lingiardi \& A Colli (Eds.), La ricerca in psicoterapia. Modelli e strumenti (pp. 67-78). Milano: Cortina.

Wolpe, J. (1958). Psychotherapy by Reciprocal Inhibition. Stanford: Stanford University Press. 
Ricerca in Psicoterapia / Research in Psychotherapy 2010; 1 (13): 61-91. http://www.researchinpsychotherapy.net

Zennaro, A., Cristofanelli, S., \& Vottero Ris, F. (2006). Indicazioni per una buona metodologia della ricerca. In N. Dazzi, V. Lingiardi \& A Colli (Eds.), La ricerca in psicoterapia. Modelli e strumenti (pp. 67-78). Milano: Cortina.

\title{
Theoretical, methodological and data-analytical issues in psychotherapy research. Part I: Process and outcome research - from the ' 50 s to the ' $80 \mathrm{~s}$
}

\begin{abstract}
Psychotherapy can be described with regard to two main dimensions: the outcome (i.e., the effects of the treatment) and the process (i.e., what is going on during the treatment). At its very beginning, the empirical investigation of psychotherapy has been mainly - although not exclusively - focused on these two distinct aspects, giving birth to what is known as psychotherapeutic outcome and process research. This period, which spans approximately from the '50s to the beginning of the ' $80 \mathrm{~s}$, represents what can be defined the classical paradigm in psychotherapy research, sustained by the aims and methodologies of neo-positivistic science. In this paper, we firstly describe the main characteristics of both outcome and process research during these three decades, with particular regard to their different research approaches and designs, levels of observation, and data analysis. Finally, we discuss what we consider to be some critical issues specific to and distinctive of each of these two distinct research domains.
\end{abstract}

Key-words: psychotherapy research; outcome research; process research; methodology; data-analysis. 
Ricerca in Psicoterapia / Research in Psychotherapy 2010; 1 (13): 61-91. http://www.researchinpsychotherapy.net 